\title{
Testing the Kerr Black Hole Hypothesis Using X-Ray Reflection Spectroscopy
}

\author{
Cosimo Bambi ${ }^{1,2}$, Alejandro Cárdenas-Avendaño ${ }^{3}$, Thomas Dauser ${ }^{4}$, Javier A. García ${ }^{5}$, and Sourabh Nampalliwar ${ }^{1}$ \\ ${ }^{1}$ Center for Field Theory and Particle Physics and Department of Physics, Fudan University, 200433 Shanghai, China; bambi@ fudan.edu.cn \\ 2 Theoretical Astrophysics, Eberhard-Karls Universität Tübingen, D-72076 Tübingen, Germany \\ ${ }^{3}$ Programa de Matemática, Fundación Universitaria Konrad Lorenz, 110231 Bogotá, Colombia \\ ${ }^{4}$ Remeis Observatory \& ECAP, Universität Erlangen-Nürnberg, D-96049 Bamberg, Germany \\ ${ }^{5}$ Harvard-Smithsonian Center for Astrophysics, Cambridge, MA 02138, USA \\ Received 2016 July 5; revised 2017 April 19; accepted 2017 May 19; published 2017 June 15
}

\begin{abstract}
We present the first X-ray reflection model for testing the assumption that the metric of astrophysical black holes is described by the Kerr solution. We employ the formalism of the transfer function proposed by Cunningham. The calculations of the reflection spectrum of a thin accretion disk are split into two parts: the calculation of the transfer function and the calculation of the local spectrum at any emission point in the disk. The transfer function only depends on the background metric and takes into account all the relativistic effects (gravitational redshift, Doppler boosting, and light bending). Our code computes the transfer function for a spacetime described by the Johannsen metric and can easily be extended to any stationary, axisymmetric, and asymptotically flat spacetime. Transfer functions and single line shapes in the Kerr metric are compared to those calculated from existing codes to check that we reach the necessary accuracy. We also simulate some observations with NuSTAR and LAD/eXTP and fit the data with our new model to show the potential capabilities of current and future observations to constrain possible deviations from the Kerr metric.
\end{abstract}

Key words: accretion, accretion disks - black hole physics - gravitation

\section{Introduction}

The theory of general relativity was proposed by Einstein about a century ago and is still the standard framework for the description of the gravitational field and the chrono-geometrical structure of the spacetime. The first test of general relativity can be dated back to the measurement of light bending by the Sun by Eddington in 1919 (Dyson et al. 1920). In particular, over the past 60 years, there have been significant efforts to test the theory in weak gravitational fields, mainly with precise experiments in the solar system and accurate radio observations of binary pulsars (Will 2014). Tests of general relativity in the strong gravity regime are nowadays the new frontier, both with electromagnetic radiation (Bambi et al. 2016; Johannsen 2016; Bambi 2017) and gravitational waves (Yunes \& Siemens 2013; Yagi \& Stein 2016).

Astrophysical black holes are the ideal laboratory for testing strong gravity. In four-dimensional general relativity, an uncharged black hole is described by the Kerr solution ${ }^{6}$ and is completely described by only two parameters, namely the mass $M$ and the spin angular momentum $J$ of the object. This is the result of the "no-hair theorem" (Carter 1971; Robinson 1975). It is remarkable that the spacetime around astrophysical black holes should be well described by the Kerr metric. As soon as a black hole is formed, initial deviations from the Kerr solution are quickly radiated away with the emission of gravitational waves (Price 1972). The equilibrium electric charge is extremely small for macroscopic objects and completely negligible for the spacetime geometry (Bambi et al. 2009). Accretion disks typically have a mass of several orders of magnitude smaller

\footnotetext{
6 There are a number of assumptions behind this statement. In particular, the spacetime must have four dimensions and be stationary and asymptotically flat; the exterior must be regular (no singularities or closed time-like curves); the metric is a vacuum solution of the Einstein equations. See, e.g., Chruściel et al (2012) for more details.
}

than the central object and their impact on the background metric can be safely ignored (Bambi et al. 2014; Barausse et al. 2014).

Within Einstein's theory of gravity, the Kerr metric should well describe the spacetime around astrophysical black holes. Nevertheless, macroscopic deviations from the Kerr spacetime are possible in many scenarios. For instance, Herdeiro \& Radu (2014) have recently discovered a family of hairy black holes in four-dimensional Einstein gravity minimally coupled to a complex, massive scalar field. Hairy black holes generically arise when scalar fields are non-minimally coupled to gravity, and an example is the dilaton in Einstein-dilaton-GaussBonnet gravity (Mignemi \& Stewart 1993). Quantum gravity effects might also produce macroscopic corrections to the Kerr metric (Dvali \& Gomez 2013a, 2013b; Giddings 2014).

Electromagnetic and gravitational radiations can test general relativity in different ways. The properties of the electromagnetic radiation emitted by the accreting gas close to a black hole depend on both the gas motion in the strong gravity region and the photon propagation from the emission point in the disk to the detection point in the flat faraway region. In this case, we can test the Kerr metric in the same way as solar system experiments have so far tested the Schwarzschild solution in the weak field limit. However, it is not possible to distinguish a Kerr black hole in general relativity from a Kerr black hole in an alternative metric theory of gravity, because the geodesic motion is the same (Psaltis et al. 2008). Gravitational waves can instead probe the field equations of the theory, while they are less suitable to perform model-independent tests. The two approaches can thus be seen as complementary; see, e.g., Konoplya \& Zhidenko (2016), Cárdenas-Avendaño et al. (2016), and Bambi \& Nampalliwar (2016).

With the electromagnetic approach, there are currently two leading techniques to probe the strong gravity region around a black hole: the study of the thermal spectrum of thin disks (continuum-fitting method; Zhang et al. 1997; Shafee et al. 2006; 
McClintock et al. 2014) and the analysis of the relativistically smeared reflection spectrum of thin disks (reflection method; Fabian et al. 1989; Brenneman \& Reynolds 2006; Reynolds 2014). Both techniques have been developed for measuring black hole spins under the assumption of Kerr background and can be naturally extended for testing the Kerr metric (Torres 2002; Lu \& Torres 2003; Schee \& Stuchlík 2009; Bambi \& Barausse 2011; Bambi 2013a, 2013b; Bambi \& Malafarina 2013; Johannsen \& Psaltis 2013; Kong et al. 2014; Ni et al. 2016).

The reflection method has a number of advantages with respect to the study of the thermal spectrum. It can be easily applied to both stellar-mass and supermassive black holes. ${ }^{7}$ It is independent of the black hole mass and distance, while the inclination angle of the disk with respect to the line of sight of the observer can be inferred from the fit of the reflection spectrum; with the continuum-fitting method, these three quantities have to be obtained from other measurements and their uncertainty is often large. In the presence of high quality data and the correct astrophysical model, the reflection method is potentially quite a powerful tool to constrain the metric around black holes (see, for instance, Jiang et al. 2015a, 2015b, 2016; Cárdenas-Avendaño et al. 2016).

Theoretical models of X-ray reflection have been undergoing active development over the past three decades (see Fabian \& Ross 2010 for a review). Currently, the most advanced model is XILLVER (García \& Kallman 2010; García et al. 2013), and its relativistic counterpart RELXILL (Dauser et al. 2013; García et al. 2014). These are state of the art in modeling reflection in strong gravity.

Compared to all earlier reflection codes, XILLVER provides a superior treatment of the radiative transfer, as well as an improved calculation of the ionization balance, by implementing the photoionization routines from the XSTAR code (Kallman \& Bautista 2001), which incorporates the most complete atomic database for modeling synthetic photoionized X-ray spectra. The microphysics captured by XILLVER is much more rigorous than for any earlier code, principally because of the detailed treatment of the K-shell atomic properties of the prominent ions (e.g., Kallman et al. 2004; García et al. 2005, 2009).

The model RELXILL is the result from the combination of XILLVER with the relativistic blurring code RELCONV (Dauser et al. 2010). RELCONV is a relativistic convolution code that, assuming the Kerr metric, requires as input the local spectrum at any emission point in the disk and gives as output the spectrum measured by a distant observer. The aim of our work here is to construct a model to extend RELXILL to a generic stationary, axisymmetric, and asymptotically flat black hole metric. We replace RELCONV with a more general relativistic convolution code, while we maintain XILLVER because the microphysics of the local spectrum does not change.

In this paper, we present a new code to compute transfer functions in any stationary, axisymmetric, and asymptotically flat black hole metric and extend RELXILL for testing the Kerr black hole hypothesis. Current studies along this line of research model the X-ray spectrum with a simple power law plus a relativistically broadened iron line (Jiang et al. 2015a, 2015b, 2016). This can be sufficient for a preliminary study and a qualitative analysis. However, this is definitively not adequate if we really want to test general

\footnotetext{
7 The continuum-fitting method has also been applied to supermassive black holes, but only in very special cases (e.g., Czerny et al. 2011; Done et al. 2013).
}

relativity. Here we employ the formalism of the transfer function for thin accretion disks (Cunningham 1975). In this framework, the calculations of the reflection spectrum are split into two parts: the calculation of the transfer function and the calculation of the reflection spectrum in the rest frame of the gas. The transfer function only depends on the metric of the background and takes into account all the relativistic effects (gravitational redshift, Doppler boosting, light bending). The local spectrum is obtained by solving radiation transfer on a plane-parallel, one-dimensional slab and is not strictly related to the metric of the spacetime.

In order to test the Kerr metric, our model must be able to compute the X-ray reflection spectrum of a thin disk in a background that is more general than the Kerr solution and that includes the Kerr solution as a special case. The test-metric is described by the mass $M$ and the spin angular momentum $J$ of the object, as well as by a number of "deformation parameters." The latter are used to quantify possible deviations from the Kerr metric and are the parameters to constrain from observations to verify the Kerr black hole hypothesis. The Kerr metric is recovered when all the deformation parameters vanish, while there are deviations from the Kerr solution in the presence of at least one non-vanishing deformation parameter.

In the standard case of the Kerr metric, the calculations of the transfer function exploit some specific properties of the Kerr solution (Cunningham 1975; Speith et al. 1995). Because of the presence of the Carter constant, the equations of motion are separable. More importantly, the equations in the $(r, \theta)$ plane can be reduced to elliptic integrals. This significantly simplifies the calculations of the transfer function. In our more general case, the transfer function is evaluated by integrating the photon geodesic equations from the point of detection in the plane of the distant observer backward in time to the point of emission in the accretion disk. Our calculations are inevitably longer than those in the Kerr metric that solve elliptic integrals.

The paper is organized as follows. In Section 2, we review the formalism of the transfer function and, in Section 3, the Johannsen metric (Johannsen 2013), which is the one adopted in our current version of the code. Section 4 describes our numerical method to compute the transfer function. In Section 5, we compare transfer functions and single iron line shapes produced by our code for a few Kerr solutions with those calculated by existing codes. Section 6 shows some examples of transfer functions and single line shapes in the Johannsen metric. In Section 7, we simulate several observations of a bright black hole binary with NuSTAR and LAD/ eXTP and we fit the data with our new version of RELXILL to constrain one of the deformation parameters in the Johannsen metric as an illustrative example of the application of the new model and the constraining power of current and future X-ray missions. Summary and conclusions are reported in Section 8. In the Appendix, we present all the formulas to compute the transfer function for a thin accretion disk in a generic stationary, axisymmetric, and asymptotically flat black hole spacetime. Throughout the paper, we employ units in which $G_{\mathrm{N}}=c=1$ and the convention of a metric with signature $(-+++)$. In Section 3, we explicitly show the black hole mass parameter $M$ as defined in the Kerr and Johannsen metrics, while in the rest of the paper we set $M=1$.

\section{Transfer Function for Thin Accretion Disks}

In this section, we review the formalism of the transfer function for geometrically thin and optically thick accretion 
disks (Cunningham 1975; Speith et al. 1995). The observed flux of a thin accretion disk (measured, for instance, in erg s ${ }^{-1} \mathrm{~cm}^{-2} \mathrm{~Hz}^{-1}$ ) can be written as

$$
F_{\mathrm{o}}\left(\nu_{\mathrm{o}}\right)=\int I_{\mathrm{o}}\left(\nu_{\mathrm{o}}, X, Y\right) d \tilde{\Omega}=\int g^{3} I_{\mathrm{e}}\left(\nu_{\mathrm{e}}, r_{\mathrm{e}}, \vartheta_{\mathrm{e}}\right) d \tilde{\Omega} .
$$

$I_{\mathrm{o}}$ and $I_{\mathrm{e}}$ are, respectively, the specific intensity of the radiation detected by the distant observer and the specific intensity of the radiation as measured by the emitter (for instance, in erg s${ }^{-1} \mathrm{~cm}^{-2} \mathrm{str}^{-1} \mathrm{~Hz}^{-1}$ ). $X$ and $Y$ are the Cartesian coordinates of the image of the disk in the plane of the distant observer. $d \tilde{\Omega}=d X d Y / D^{2}$ is the element of the solid angle subtended by the image of the disk in the observer's sky and $D$ is the distance of the observer from the source. $I_{0}=g^{3} I_{\mathrm{e}}$ follows from Liouville's theorem, where $g=\nu_{\mathrm{o}} / \nu_{\mathrm{e}}$ is the redshift factor, $\nu_{\mathrm{o}}$ is the photon frequency as measured by the distant observer, and $\nu_{\mathrm{e}}$ is the photon frequency in the rest frame of the emitter. $r_{\mathrm{e}}$ is the emission radius in the disk and $\vartheta_{\mathrm{e}}$ is the emission angle (which can be different from the viewing angle of the observer $i$ because of the effect of light bending).

Introducing the transfer function $f$, the observed flux can be rewritten as

$$
\begin{aligned}
F_{\mathrm{o}}\left(\nu_{\mathrm{o}}\right)= & \frac{1}{D^{2}} \int_{r_{\text {in }}}^{r_{\text {out }}} \int_{0}^{1} \pi r_{\mathrm{e}} \frac{g^{2}}{\sqrt{g^{*}\left(1-g^{*}\right)}} \\
& \times f\left(g^{*}, r_{\mathrm{e}}, i\right) I_{\mathrm{e}}\left(\nu_{\mathrm{e}}, r_{\mathrm{e}}, \vartheta_{\mathrm{e}}\right) d g^{*} d r_{\mathrm{e}},
\end{aligned}
$$

where $r_{\text {in }}$ and $r_{\text {out }}$ are, respectively, the inner and the outer edge of the accretion disk. In the Novikov-Thorne model (Novikov et al. 1973; Page \& Thorne 1974), the inner edge of the disk is assumed to be located at the innermost stable circular orbit (ISCO). The outer edge can be set at some large radius where the emission becomes negligible; in our calculation it will be located at $\sim 1000$. The expression of the transfer function $f$ is (Cunningham 1975)

$$
f\left(g^{*}, r_{\mathrm{e}}, i\right)=\frac{1}{\pi r_{\mathrm{e}}} g \sqrt{g^{*}\left(1-g^{*}\right)}\left|\frac{\partial(X, Y)}{\partial\left(g^{*}, r_{\mathrm{e}}\right)}\right|,
$$

where the relative redshift factor $g^{*}$ is defined as

$$
g^{*}=\frac{g-g_{\min }}{g_{\max }-g_{\min }},
$$

which ranges from 0 to 1 . Here $g_{\max }=g_{\max }\left(r_{\mathrm{e}}, i\right)$ and $g_{\min }=g_{\min }\left(r_{\mathrm{e}}, i\right)$ are, respectively, the maximum and the minimum values of the redshift factor $g$ for the photons emitted from the radial coordinate $r_{\mathrm{e}}$ and detected by a distant observer with polar coordinate $i .\left|\partial(X, Y) / \partial\left(g^{*}, r_{\mathrm{e}}\right)\right|$ is the Jacobian. The transfer function thus acts as an integration kernel to calculate the spectrum detected by the distant observer starting from the local spectrum at any point of the disk. Let us note that in the specific intensity $I_{\mathrm{e}}, \nu_{\mathrm{e}}$, and $\vartheta_{\mathrm{e}}$ must be written in terms of $g^{*}$ and $r_{\mathrm{e}}$. In our model, only the primary image of the accretion disk is taken into account; that is, we neglect secondary and higher order images generated by photons crossing the equatorial plane and then landing on the disk.

The transfer function $f\left(g^{*}, r_{\mathrm{e}}, i\right)$ only depends on the metric of the spacetime and the position of the distant observer. It

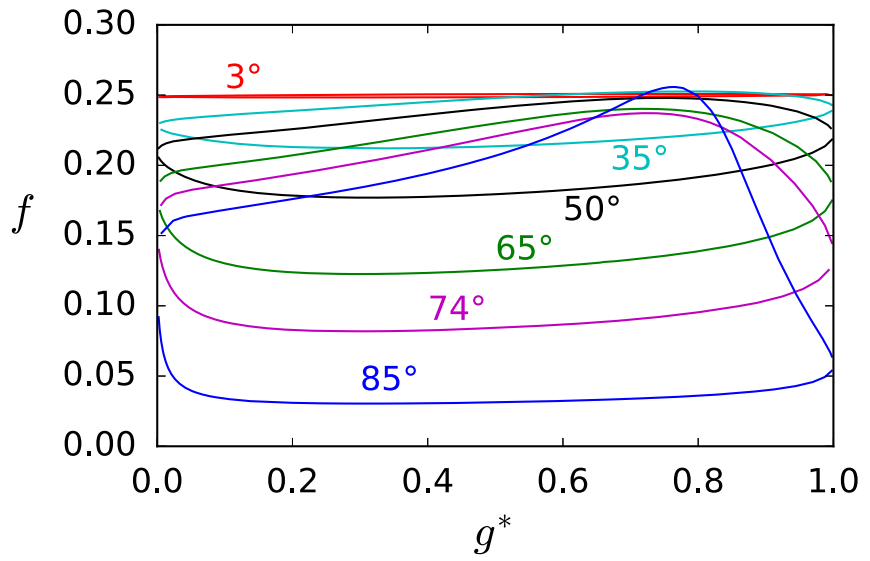

Figure 1. Impact of the viewing angle $i$ on the transfer function $f$. Here the spacetime is described by the Kerr metric with the spin parameter $a_{*}=0.998$ and the emission radius is $r_{\mathrm{e}}=4$. The values of the viewing angle are indicated.

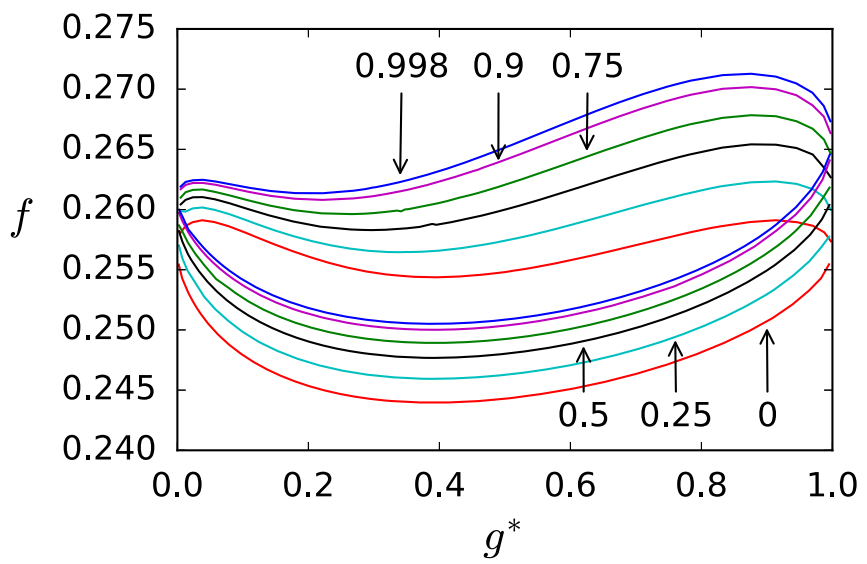

Figure 2. Impact of the dimensionless spin parameter $a_{*}$ on the transfer function $f$. Here the spacetime is described by the Kerr metric, the emission radius is $r_{\mathrm{e}}=7$, and the viewing angle is $i=30^{\circ}$. The values of the spin parameter are indicated.

takes into account all the relativistic effects (gravitational redshift, Doppler boosting, light bending). For a fixed emission radius $r_{\mathrm{e}}$ and viewing angle $i$, the transfer function is a closed curve parameterized by $g^{*}$, see Figures 1 and 2 . This is true except in the special cases $i=0$ and $\pi / 2$. There is only one point in the disk for which $g^{*}=1$ and only one point for which $g^{*}=0$. These points are connected by two curves, so we have two branches of the transfer function, say $f^{(1)}\left(g^{*}, r_{\mathrm{e}}, i\right)$ and $f^{(2)}\left(g^{*}, r_{\mathrm{e}}, i\right)$. In the case of isotropic emission $\left(I_{\mathrm{e}}\right.$ independent of $\vartheta_{\mathrm{e}}$ and of the emission azimuthal angle) in an axisymmetric system (e.g., no orbiting spots), Equation (2) can be written as

$$
\begin{aligned}
F_{\mathrm{o}}\left(\nu_{\mathrm{o}}\right)= & \frac{1}{D^{2}} \int_{r_{\text {in }}}^{r_{\text {out }}} \int_{0}^{1} \frac{\pi r_{\mathrm{e}} g^{2}}{\sqrt{g^{*}\left(1-g^{*}\right)}} \\
& \times\left[f^{(1)}\left(g^{*}, r_{\mathrm{e}}, i\right)+f^{(2)}\left(g^{*}, r_{\mathrm{e}}, i\right)\right] I_{\mathrm{e}}\left(\nu_{\mathrm{e}}, r_{\mathrm{e}}\right) d g^{*} d r_{\mathrm{e}} .
\end{aligned}
$$

If $I_{\mathrm{e}}$ does depend on $\vartheta_{\mathrm{e}}$, it is necessary to perform the integral twice, one for the upper branch, one for the lower one, so 
Equation (2) becomes

$$
\begin{aligned}
F_{\mathrm{o}}\left(\nu_{\mathrm{o}}\right)= & \frac{1}{D^{2}} \int_{r_{\text {in }}}^{r_{\text {out }}} \int_{0}^{1} \frac{\pi r_{\mathrm{e}} g^{2}}{\sqrt{g^{*}\left(1-g^{*}\right)}} \\
& \times f^{(1)}\left(g^{*}, r_{\mathrm{e}}, i\right) I_{\mathrm{e}}\left(\nu_{\mathrm{e}}, r_{\mathrm{e}}, \vartheta_{\mathrm{e}}^{(1)}\right) d g^{*} d r_{\mathrm{e}} \\
& +\frac{1}{D^{2}} \int_{r_{\text {in }}}^{r_{\text {out }}} \int_{0}^{1} \frac{\pi r_{\mathrm{e}} g^{2}}{\sqrt{g^{*}\left(1-g^{*}\right)}} \\
& \times f^{(2)}\left(g^{*}, r_{\mathrm{e}}, i\right) I_{\mathrm{e}}\left(\nu_{\mathrm{e}}, r_{\mathrm{e}}, \vartheta_{\mathrm{e}}^{(2)}\right) d g^{*} d r_{\mathrm{e}},
\end{aligned}
$$

where $\vartheta_{\mathrm{e}}^{(1)}$ and $\vartheta_{\mathrm{e}}^{(2)}$ indicate the emission angles with relative redshift factor $g^{*}$, respectively, in the branches 1 and 2 .

\section{Non-Kerr Model}

Model-independent tests of the Kerr metric can be performed by adopting a background that is more general than the Kerr solution and that includes the Kerr solution as a special case. In addition to the mass $M$ and the spin angular momentum $J$, the metric has a number of deformation parameters used to quantify possible deviations from the Kerr spacetime. The values of these deformation parameters can be constrained by observations. If astrophysical black holes are Kerr black holes, observations should require vanishing deformation parameters. If observations require that at least one of the deformation parameters is non-vanishing, this may be interpreted as an indication of the presence of new physics.

Our current code adopts the Johannsen metric with four deformation parameters (Johannsen 2013). In Boyer-Lindquist coordinates, the line element reads

$$
\begin{aligned}
d s^{2}= & -\frac{\tilde{\Sigma}\left(\Delta-a^{2} A_{2}^{2} \sin ^{2} \theta\right)}{B^{2}} d t^{2} \\
& -\frac{2 a\left[\left(r^{2}+a^{2}\right) A_{1} A_{2}-\Delta\right] \tilde{\Sigma} \sin ^{2} \theta}{B^{2}} \\
& \times d t d \phi+\frac{\tilde{\Sigma}}{\Delta A_{5}} d r^{2}+\tilde{\Sigma} d \theta^{2} \\
& +\frac{\left[\left(r^{2}+a^{2}\right)^{2} A_{1}^{2}-a^{2} \Delta \sin ^{2} \theta\right] \tilde{\Sigma} \sin ^{2} \theta}{B^{2}} d \phi^{2},
\end{aligned}
$$

where $a=J / M$,

$$
\begin{aligned}
& B=\left(r^{2}+a^{2}\right) A_{1}-a^{2} A_{2} \sin ^{2} \theta, \quad \tilde{\Sigma}=\Sigma+f, \\
& \Sigma=r^{2}+a^{2} \cos ^{2} \theta, \quad \Delta=r^{2}-2 M r+a^{2},
\end{aligned}
$$

and

$$
\begin{aligned}
f & =\epsilon_{3} \frac{M^{3}}{r}, \quad A_{1}=1+\alpha_{13}\left(\frac{M}{r}\right)^{3}, \\
A_{2} & =1+\alpha_{22}\left(\frac{M}{r}\right)^{2}, \quad A_{5}=1+\alpha_{52}\left(\frac{M}{r}\right)^{2} .
\end{aligned}
$$

The deformation parameters are $\epsilon_{3}, \alpha_{13}, \alpha_{22}$, and $\alpha_{52}$ and are dimensionless. Such a metric has the correct Newtonian limit and is consistent with the current PPN constraints (Johannsen 2013). It exactly reduces to the Kerr metric for $\epsilon_{3}=\alpha_{13}=\alpha_{22}=$ $\alpha_{52}=0$.

The Johannsen metric also has a Carter-like constant. The normal of the disk at the point of emission becomes (see the
Appendix for more details)

$$
n^{\mu}=\left[0,0,\left(r_{\mathrm{e}}^{2}+\epsilon_{3} \frac{M^{3}}{r_{\mathrm{e}}}\right)^{-1 / 2}, 0\right] .
$$

The emission angle $\vartheta_{\mathrm{e}}$ can now be written as

$$
\cos \vartheta_{\mathrm{e}}=q g\left(r_{\mathrm{e}}^{2}+\epsilon_{3} \frac{M^{3}}{r_{\mathrm{e}}}\right)^{-1 / 2},
$$

where $q^{2}=\mathcal{Q} / E^{2}, \mathcal{Q}$ is the Carter-like constant of the photon, and $E$ is the photon energy. In the Johannsen metric, the Carterlike constant has the same form as the Carter constant in the Kerr metric even for non-vanishing deformation parameters (Johannsen 2014), and $k_{\theta}=q k_{t}$ when the photon hits the disk in the equatorial plane. $q$ can be inferred from the photon initial conditions (as in the Kerr metric)

$$
X_{0}=\frac{\lambda}{\sin i}, \quad Y_{0}=\sqrt{q^{2}+a^{2} \cos ^{2} i-\lambda^{2} \cot ^{2} i},
$$

where $\lambda=L_{z} / E$.

In the Kerr metric, we have an exterior regular spacetime for $|a| \leqslant M$, which is the condition for the existence of an event horizon. For $|a|>M$, the spacetime has a naked singularity. In the Johannsen metric, if we require a regular exterior region (no singularities or closed time-like curves) we have the following conditions on the deformation parameters (Johannsen 2013)

$$
\begin{gathered}
\alpha_{13}, \epsilon_{3} \geqslant-\left(\frac{M+\sqrt{M^{2}-a^{2}}}{M}\right)^{3}, \\
\alpha_{22}, \alpha_{25} \geqslant-\left(\frac{M+\sqrt{M^{2}-a^{2}}}{M}\right)^{2} .
\end{gathered}
$$

We impose these conditions on the deformation parameters in order to avoid spacetimes with pathological properties.

\section{Numerical Method}

In this section, we describe our algorithm for calculating the transfer function and creating the Master Table that is used to construct the model for RELCONV. The Master Table has data in three dimensions: spin, deformation parameter, and inclination angle. The grid sizes along each dimension are 30, 30, and 22 , respectively. The points along the spin and inclination angle grids are non-uniform and independent of each other. The points along the deformation parameter grid depend on the spin: the points are chosen such that the ISCO radii at each spin, for the range of deformation parameters at that spin, span the range from the minimum Kerr ISCO radius to the maximum Kerr ISCO radius. Figure 3 shows these grid points for the deformation parameter $\alpha_{13}$.

At each configuration (namely a grid point with a specific spin, deformation parameter, and inclination angle), we discretize the accretion disk with a grid of 100 emission radii $r_{\mathrm{e}}$ and at each emission radius we tabulate the transfer function at 20 equally spaced values of $g^{*}$ on each branch. ${ }^{8}$ The scheme for choosing emission radii and $g^{*}$ and the values of spin and inclination angles along the grid are the same as those used in

\footnotetext{
$8 g^{*}=0$ and $g^{*}=1$ are replaced, respectively, by $g^{*}=0.002$ and $g^{*}=0.998$ for numerical reasons, because the Jacobian diverges at $g^{*}=0$ and 1 .
} 


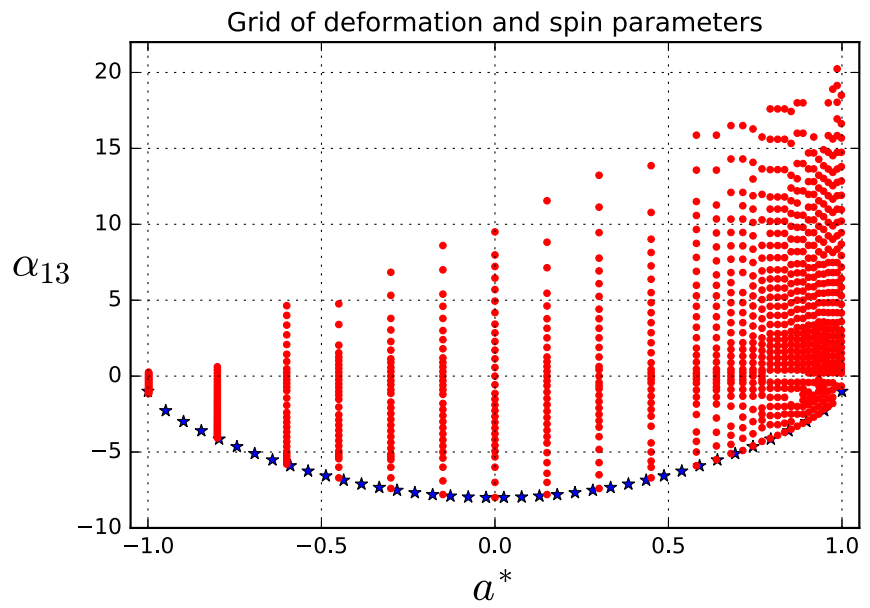

Figure 3. Points of the grid of the Master Table for the spin parameter $a_{*}$ and the deformation parameter $\alpha_{13}$.

the standard RELCONV model. The resulting Master Table has sufficient resolution such that the transfer functions at arbitrary configurations can be interpolated accurately.

Notice that the choice of the range of the deformation parameters is somewhat arbitrary. If we adopt the point of view that deviations from the Kerr metric must be small and that the deformation parameters in Equation (9) are the leading order terms in an expansion, these deformation parameters must also be small and we may restrict the attention to the ranges of $(-1,1)$. However, here we adopt the same spirit as Johannsen (2014); we do not impose that the deformation parameters must be small quantities. Since the impact of each deformation parameter on the reflection spectrum is different (see Section 6), it is convenient to adopt different ranges for different deformation parameters.

Before describing each step in detail, we draw an outline of the code. The first step to calculate the transfer function table for each configuration, i.e., for specific values of $\left(a_{*}, \epsilon_{3}, \alpha_{13}\right.$, $\left.\alpha_{22}, \alpha_{52}, i\right)$, is to determine the radius of the ISCO. After that, we determine 100 values of the emission radius $r_{\mathrm{e}}$ at which we will evaluate the transfer function. We then consider an observer at the distance $D=10^{8} M$, so that the spacetime near the observer can be assumed to be flat. We create a grid in the observer plane and, from each point of this grid, we fire photons and calculate their trajectories backward in time from the point of detection in the image plane to the point of emission on the disk. We adjust the position of the photons in the grid adaptively such that they arrive precisely at the radius $r_{\mathrm{e}}$ of interest in the accretion disk. We denote by central photon each photon that hits the accretion disk at one of the target emission radii. We then evaluate the redshift factor $g$ and the emission angle $\vartheta_{\mathrm{e}}$ for each central photon. At this point, we fire four photons in a small grid around the central photon and evaluate the Jacobian at each central photon. The size of this small grid is chosen to ensure that the value of the Jacobian has converged and does not change for any smaller grid size. We also use an adaptive method to determine the minimum and maximum values of the redshift factor for each radius $r_{\mathrm{e}}$. After finding enough central photons to produce a transfer function curve, we split the central photons into two branches according to their position on the grid relative to the photons associated with the minimum and the maximum redshift factors. Subsequently, we calculate $g^{*}$ and the transfer function, as defined in Equation (3) at each central photon. Since the transfer function at each branch is evaluated at 20 equally spaced values of $g^{*}$, we interpolate our transfer functions as a function of $g^{*}$ and obtain their values at the requisite $g^{*}$ values. This is performed for each of the 100 emission radii of interest. We repeat this process for any configuration $\left(a_{*}, \epsilon_{3}, \alpha_{13}, \alpha_{22}\right.$, $\left.\alpha_{52}, i\right)$.

The grid of the plane of the distant observer is adaptive, based on a standard elliptical grid. The points in the grid are defined as

$$
\begin{aligned}
X_{0}(r, \phi) & =r \cos \phi, \\
Y_{0}(r, \phi) & =r \sin \phi \cos i,
\end{aligned}
$$

where $i$ is again the inclination angle of the disk with respect to the line of sight of the distant observer and

$$
\begin{gathered}
\phi=\frac{2 \pi}{N} j \quad j=\{1,2, \ldots, N\}, \\
r=\left(r_{\mathrm{e}}\right)_{k} \quad k=\{1,2, \ldots, 100\} .
\end{gathered}
$$

$N$ is chosen to be 61 . The photon trajectories are calculated from the image plane of the distant observer to the emission point in the disk by solving the geodesic equations with the ray-tracing code of Bambi (2012), which employs an adaptive step-size fourth-order Runge-Kutta-Nyström algorithm (Lund et al. 2009). The Christoffel symbols appearing in the geodesic equations are evaluated from their analytical formulas, which have been implemented in the code. Due to gravitational bending, the initial grid of photons does not always hit the accretion disk at the requisite radii. Therefore, the code adjusts $r$ until the photon hits the accretion disk at the emission radius of interest with a precision of $10^{-6}$.

Around each central photon, we choose four photons, for which the location on the observer grid relative to the central photon is $\left(X_{0} \pm \Delta X, Y_{0} \pm \Delta Y\right)$, where

$$
\Delta X=10^{-4}+10^{-4} X_{0}, \quad \Delta Y=10^{-4}+10^{-4} Y_{0} .
$$

The Jacobian in the expression of the transfer function is calculated at each central photon from

$$
\left|\frac{\partial(X, Y)}{\partial\left(g^{*}, r_{\mathrm{e}}\right)}\right|=\left|\frac{\partial r_{\mathrm{e}}}{\partial X} \frac{\partial g^{*}}{\partial Y}-\frac{\partial r_{\mathrm{e}}}{\partial Y} \frac{\partial g^{*}}{\partial X}\right|^{-1} .
$$

Using the preliminary $\phi$ grid defined in Equation (15), we find the minimum and maximum redshift factor, $g_{\min }$ and $g_{\text {max }}$, respectively, for any specific emission radius $r_{\mathrm{e}}$. While evaluating the central photons along the $\phi$ grid, we record that central photon as our preliminary $g_{\min }\left(g_{\max }\right)$, which has the smallest (largest) redshift factor among the central photons on the grid. We then evaluate the redshift factor on either side of these preliminary extrema with adaptive step-size to move toward the actual $g_{\min }\left(g_{\max }\right)$. For each of the extrema, when the change in redshift factor between two consecutive steps is below $10^{-6}$, we assign that central photon as describing those extrema. Using $g_{\min }$ and $g_{\max }$, we then calculate $g^{*}$ at every other central photon. Let us denote the $\phi$ values corresponding to $g_{\min }$ and $g_{\max }$ as $\phi_{\min }$ and $\phi_{\max }$, respectively. $\phi_{\min }$ and $\phi_{\max }$ then divide the whole range of $\phi$ (from 0 to $2 \pi$ ) into two branches:

$$
\phi_{\min }<\phi<\phi_{\max }
$$


and

$$
\phi_{\min }>\phi>\phi_{\max }
$$

Due to strong gravitational bending, especially at emission radii near the ISCO, it may happen that $\phi_{\min }$ and $\phi_{\max }$ are close to each other. In this case, the preliminary $\phi$ grid is unable to provide enough central photons on both branches. Consequently, an interpolation of the transfer function can perform poorly. To avoid this, we compare $g^{*}$ for each consecutive pair of central photons on the initial $\phi$ grid. If the difference between consecutive $g^{*}$, $\mathrm{s}$ is larger than 0.05 , we find additional central photons between the two, such that there are enough $g^{*}$ to obtain a good interpolation.

The above procedure is repeated for each configuration $\left(a_{*}\right.$, $\left.\epsilon_{3}, \alpha_{13}, \alpha_{22}, \alpha_{52}, i\right)$. The data obtained are then fed into a Python routine. For each configuration and each emission radius $r_{\mathrm{e}}$, the Python routine splits the data into two branches according to Equations (19) and (20), performs a linear interpolation, and generates a pair of transfer functions at constantly spaced $g^{*}$. Additionally, the emission angles at central photons are also interpolated in the same way to obtain their values at the requisite $g^{*}$. For each configuration, the data, which comprise of the values of $r_{\mathrm{e}}, g_{\min }, g_{\max }$, transfer functions, and emission angles $\vartheta_{\mathrm{e}}$, are stored in a list, and a FITS file (Master Table) is generated with all the configurations.

\section{Comparison with Existing Codes for the Kerr Metric}

In this section, we want to test if the line shapes and transfer functions produced by the code discussed in this paper agree with existing simulations for the Kerr case. We will use the RELLINE model to incorporate the non-Kerr relativistic smearing of the reflection spectrum and therefore this model is also used for comparison. The very good agreement of the RELLINE model with other existing model codes has been shown previously (see Dauser et al. 2010).

The transfer functions and additional information of the raytracing simulations are stored in a table, which is in the same format as the table used by the RELLINE model. This allows us to use the RELLINE model code to predict the line shape for the non-Kerr spacetimes and directly compare the calculated shapes and transfer functions for the Kerr case.

Table 1 shows the values of the transfer functions from our code and from RELLINE for the Kerr metric with the spin parameter $a_{*}=0.9982$ and the cosine of the viewing angle $\mu=0.3221819$ (viewing angle $i=71^{\circ} .21$ ). For illustration, we report three emission radii, namely $r_{\mathrm{e}}=1.2468,4.7197$, and 41.309 , and five relative redshift factors $g^{*}$. The actual values of the redshift factor, $g$, as computed by the two codes, are also shown. For every $r_{\mathrm{e}}$ and $g^{*}$, there are two values of the transfer function; the first line refers to the values of the transfer function in the first branch, $f^{(1)}$ in Equation (6), the second line to the values of the transfer function in the second branch, $f^{(2)}$. Table 2 shows the values of the emission angles, $\vartheta_{\mathrm{e}}^{(1)}$ and $\vartheta_{\mathrm{e}}^{(2)}$, for the same configuration. Tables 3 and 4 report the transfer functions and the cosines of the emission angles for the Kerr metric with $a_{*}=-0.45$ and $\mu=0.8622873$ (viewing angle $i=30^{\circ} .43$ ).

In Figure 4, we compare the relativistic line between our code and RELLINE for a few representative cases. In the top panels, we have a fast-rotating black hole with spin parameter $a_{*}=0.998$. In the bottom panels, we have the iron line from a retrograde disk and the black hole spin is $a_{*}=-0.5$. In the left
Table 1

Transfer function: comparison with RELLINE

\begin{tabular}{|c|c|c|c|c|c|c|}
\hline$r_{\mathrm{e}}=1.2468$ & & 2 & 7 & $\begin{array}{l}g^{*} \\
11\end{array}$ & 15 & 19 \\
\hline \multirow[t]{3}{*}{ This work } & $g$ & 0.06573 & 0.19584 & 0.29993 & 0.40401 & 0.50810 \\
\hline & $f^{(1)}$ & 0.10682 & 0.12469 & 0.12479 & 0.11934 & 0.10365 \\
\hline & $f^{(2)}$ & 0.02651 & 0.02935 & 0.03863 & 0.05128 & 0.07216 \\
\hline \multirow[t]{3}{*}{ RELLINE } & $g$ & 0.06571 & 0.19568 & 0.29965 & 0.40363 & 0.50761 \\
\hline & $f^{(1)}$ & 0.10729 & 0.12462 & 0.12473 & 0.11897 & 0.10385 \\
\hline & $f^{(2)}$ & 0.02639 & 0.02929 & 0.03862 & 0.05122 & 0.07188 \\
\hline \multicolumn{2}{|l|}{$r_{\mathrm{e}}=4.7197$} & 2 & 7 & $\begin{array}{l}g^{*} \\
11\end{array}$ & 15 & 19 \\
\hline \multirow[t]{3}{*}{ This work } & $g$ & 0.48405 & 0.71230 & 0.89491 & 1.07752 & 1.26013 \\
\hline & $f^{(1)}$ & 0.18295 & 0.19639 & 0.21602 & 0.23010 & 0.18632 \\
\hline & $f^{(2)}$ & 0.11339 & 0.09843 & 0.10058 & 0.10676 & 0.12315 \\
\hline \multirow[t]{3}{*}{ RELLINE } & $g$ & 0.48406 & 0.71229 & 0.89486 & 1.07745 & 1.26003 \\
\hline & $f^{(1)}$ & 0.18286 & 0.19636 & 0.21598 & 0.23007 & 0.18653 \\
\hline & $f^{(2)}$ & 0.11314 & 0.09837 & 0.10054 & 0.10672 & 0.12305 \\
\hline \multicolumn{2}{|l|}{$r_{\mathrm{e}}=41.309$} & 2 & 7 & $\begin{array}{l}g^{*} \\
11\end{array}$ & 15 & 19 \\
\hline \multirow[t]{3}{*}{ This work } & $g$ & 0.85331 & 0.93102 & 0.99318 & 1.05535 & 1.11751 \\
\hline & $f^{(1)}$ & 0.11711 & 0.12170 & 0.12347 & 0.13112 & 0.11257 \\
\hline & $f^{(2)}$ & 0.10404 & 0.10249 & 0.10245 & 0.10287 & 0.10442 \\
\hline \multirow[t]{3}{*}{ RELLINE } & $g$ & 0.85331 & 0.93102 & 0.99318 & 1.05535 & 1.11751 \\
\hline & $f^{(1)}$ & 0.11710 & 0.12169 & 0.12344 & 0.13129 & 0.11258 \\
\hline & $f^{(2)}$ & 0.10403 & 0.10248 & 0.10244 & 0.10287 & 0.10440 \\
\hline
\end{tabular}

Comparison between the redshift factors $g$ and the transfer functions calculated by the code described in this paper and by RELLINE at three different values of the emission radius $r_{\mathrm{e}}$ and five values of $g^{*}$ (the Master Table has 20 equally spaced values of $g^{*}$ with $g^{*}(1)=0.002$ and $g^{*}(20)=0.998$, see the text for details). These quantities are calculated for the Kerr metric with the spin parameter $a_{*}=0.9982$ and the cosine of the viewing angle $\mu=0.3221819$ (viewing angle $i \approx 71^{\circ}$ ). For every $g^{*}$, there are two values for the transfer function, corresponding, respectively, to the values of the transfer function in the upper (first line) and lower (second line) branches.

panels, the viewing angle is $i=30^{\circ}$, while it is $i=70^{\circ}$ in the right panels. The box below every panel shows the difference in percentage between the two lines. This is usually within $1 \%$. As can be seen, both line shapes are in very good agreement and therefore we conclude that the presented ray-tracing code agrees with existing model codes for calculating relativistic reflection.

As a last check, we perform a more quantitative analysis to compare the level of accuracy of our transfer functions. We simulate some observations with LAD/eXTP (Zhang et al. 2016). eXTP is a future X-ray mission and LAD will have an effective area of $3.4 \mathrm{~m}^{2}$ at $6 \mathrm{keV}$. The theoretical model is a power law plus an iron line generated by RELLINE. We consider the case of a bright binary (flux between 2 and $10 \mathrm{keV}$ at the level of $10^{-9} \mathrm{erg} \mathrm{s}^{-1} \mathrm{~cm}^{-2}$ ) and the equivalent width of the iron line is $\sim 400 \mathrm{eV}$. We adopt an exposure time of $1 \mathrm{Ms}$. All these parameters (brightness of the source, equivalent width of the iron line, exposure time) are quite optimistic, so we can obtain a good measurement. We then fit the simulated data with both our single iron lines for Kerr and with RELLINE, and we compare the difference. 
Table 2

Transfer function: comparison with RELLINE

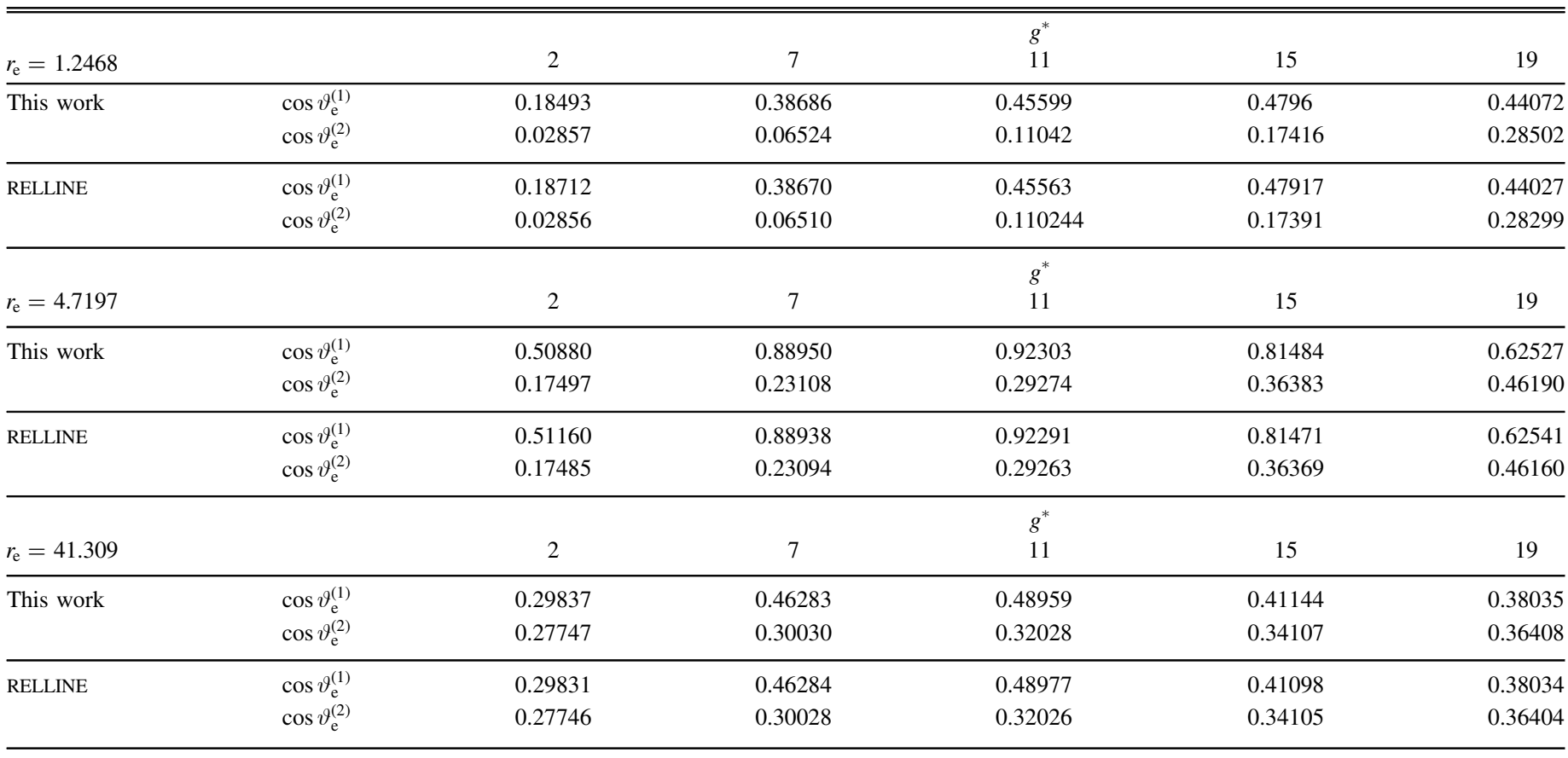

Same as in Table 1 for the cosine of the emission angle. $\vartheta_{\mathrm{e}}^{(1)}$ and $\vartheta_{\mathrm{e}}^{(2)}$ refer, respectively, to the upper and lower branches.

Table 3

Transfer function: comparison with RELLINE

\begin{tabular}{|c|c|c|c|c|c|c|}
\hline$r_{\mathrm{e}}=7.5154$ & & 2 & 7 & $\begin{array}{l}g^{*} \\
11\end{array}$ & 15 & 19 \\
\hline \multirow[t]{3}{*}{ This work } & $g$ & 0.64784 & 0.73789 & 0.80994 & 0.88199 & 0.95403 \\
\hline & $f^{(1)}$ & 0.25996 & 0.25413 & 0.25311 & 0.25484 & 0.25644 \\
\hline & $f^{(2)}$ & 0.24950 & 0.24350 & 0.24375 & 0.24604 & 0.25107 \\
\hline \multirow[t]{3}{*}{ RELLINE } & $g$ & 0.64784 & 0.73789 & 0.80994 & 0.88198 & 0.95402 \\
\hline & $f^{(1)}$ & 0.25997 & 0.25414 & 0.25309 & 0.25482 & 0.25644 \\
\hline & $f^{(2)}$ & 0.24946 & 0.24347 & 0.24372 & 0.24601 & 0.25099 \\
\hline \multicolumn{2}{|l|}{$r_{\mathrm{e}}=25.786$} & 2 & 7 & $\begin{array}{l}g^{*} \\
11\end{array}$ & 15 & 19 \\
\hline \multirow[t]{3}{*}{ This work } & $g$ & 0.86191 & 0.91369 & 0.95511 & 0.99653 & 1.03795 \\
\hline & $f^{(1)}$ & 0.27586 & 0.27202 & 0.27114 & 0.27350 & 0.27544 \\
\hline & $f^{(2)}$ & 0.27185 & 0.26957 & 0.26945 & 0.27018 & 0.27221 \\
\hline \multirow[t]{3}{*}{ RELLINE } & $g$ & 0.86191 & 0.91369 & 0.95511 & 0.99653 & 1.03795 \\
\hline & $f^{(1)}$ & 0.27587 & 0.27199 & 0.27113 & 0.27350 & 0.27545 \\
\hline & $f^{(2)}$ & 0.27184 & 0.26956 & 0.26945 & 0.27016 & 0.27226 \\
\hline \multicolumn{2}{|l|}{$r_{\mathrm{e}}=158.52$} & 2 & 7 & $\begin{array}{l}g^{*} \\
11\end{array}$ & 15 & 19 \\
\hline \multirow[t]{3}{*}{ This work } & $g$ & 0.95632 & 0.97738 & 0.99422 & 1.01106 & 1.02791 \\
\hline & $f^{(1)}$ & 0.27496 & 0.27419 & 0.27388 & 0.27449 & 0.27494 \\
\hline & $f^{(2)}$ & 0.27424 & 0.27385 & 0.27381 & 0.27391 & 0.27427 \\
\hline \multirow[t]{3}{*}{ RELLINE } & $g$ & 0.95632 & 0.97738 & 0.99422 & 1.01106 & 1.02791 \\
\hline & $f^{(1)}$ & 0.27487 & 0.27419 & 0.27388 & 0.27449 & 0.27496 \\
\hline & $f^{(2)}$ & 0.27425 & 0.27385 & 0.27381 & 0.27391 & 0.27428 \\
\hline
\end{tabular}

Same as in Table 1, but for the Kerr metric with the spin parameter $a_{*}=-0.45$ and the cosine of the viewing angle $\mu=0.8622873$ (viewing angle $i \approx 30^{\circ}$ ).
The two models provide measurements in very good agreement, suggesting that our code can compute the transfer function in the Kerr metric with the necessary precision for very accurate measurements. Figure 5 shows the result of one of our simulations. The input spin parameter and the input viewing angle of the simulations are, respectively, $a_{*}=0.9$ and $i=45^{\circ}$. When we fit the simulated data with the table of transfer functions generated by our new code, we find (here the error is at the $90 \%$ confidence level)

$$
a_{*}=0.8996 \pm 0.0008, \quad i=44^{\circ} .977 \pm 0.010 .
$$

When we use RELLINE, we obtain

$$
a_{*}=0.8997 \pm 0.0008, \quad i=44^{\circ} .992 \pm 0.010 .
$$

The difference between the two models is much smaller than what one can imagine to measure with the next generation of $\mathrm{X}$-ray satellites (and maybe even with $\mathrm{X}$-ray reflection spectroscopy in general). Figure 5 shows the contour of $\Delta \chi^{2}$. We have obtained similar results with different input parameters.

\section{Single Line Shapes in Non-Kerr Spacetimes}

Unlike existing codes for the Kerr metric, our transfer function code uses formulas valid for any stationary, axisymmetric, and asymptotically flat black hole spacetime. It is sufficient to set the deformation parameters to a non-vanishing value to obtain the corresponding transfer function and single line shape.

Examples of transfer functions in the Johannsen metric are shown in Figure 6. Each panel shows the impact of one of the deformation parameters on the transfer function, assuming that the other three deformation parameters vanish. All the transfer 
Table 4

Transfer function: comparison with RELLINE

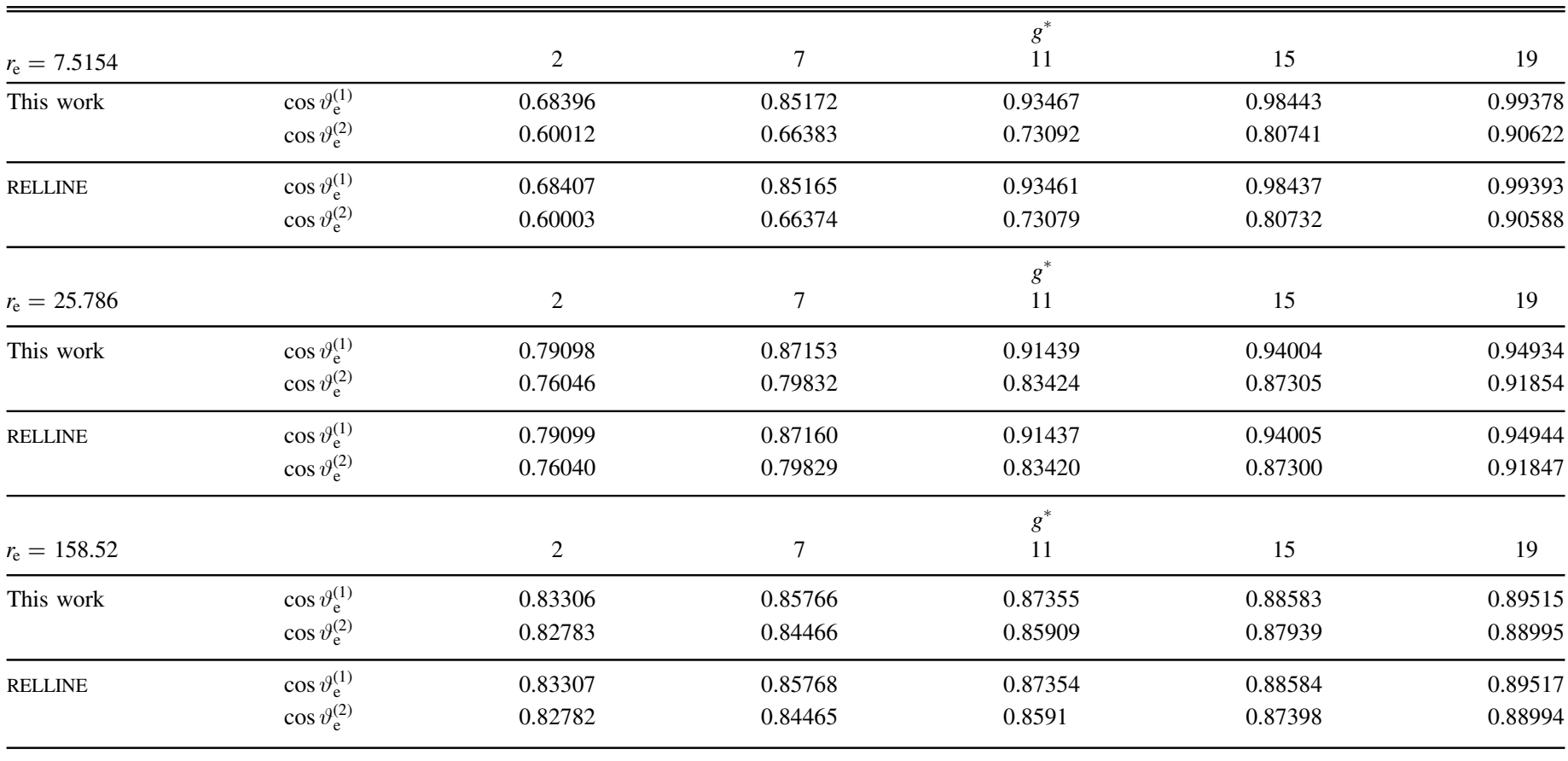

Same as in Table 2, but for the Kerr metric with the spin parameter $a_{*}=-0.45$ and the cosine of the viewing angle $\mu=0.8622873\left(\right.$ viewing angle $i \approx 30^{\circ}$ ).

functions have been evaluated at the emission radius $r_{\mathrm{e}}=6.855$, for a viewing angle $i=30^{\circ}$, and for a spin parameter $a_{*}=0.8$. The transfer function for the Kerr metric with $\epsilon_{3}=\alpha_{13}=\alpha_{22}=\alpha_{52}=0$ is the black solid curve. The other curves correspond to the transfer functions for $\epsilon_{3}= \pm 1$ and \pm 2 (top left panel), $\alpha_{13}= \pm 1$ and \pm 2 (top right panel), $\alpha_{22}= \pm 1$ and \pm 2 (bottom left panel), and $\alpha_{52}= \pm 1$ and \pm 2 (bottom right panel).

The single iron line shapes of the spacetimes considered in Figure 6 are shown in Figure 7. The emission line is at $E_{\mathrm{e}}=6.4 \mathrm{keV}$. The inner edge of the disk is set at the ISCO radius, while the other edge is at $r_{\text {out }}=400$. The local spectrum $I_{\mathrm{e}}$ is modeled with a power law with an emissivity index equal to 3 , namely $I_{\mathrm{e}} \propto 1 / r_{\mathrm{e}}^{3}$. As already discussed in Johannsen (2013), $\alpha_{13}$ and $\alpha_{22}$ strongly affect the ISCO radius and the iron line shape, $\epsilon_{3}$ has a moderate impact on both the ISCO radius and the iron line shape, while $\alpha_{52}$ does not affect the ISCO radius and has an extremely weak impact on the iron line shape.

In Figure 7, the maximum energy of the line does not change with the value of the deformation parameter. For $i=30^{\circ}$, the Doppler effect is moderate, and the photons with the highest energies come from relatively large radii $\left(r_{\mathrm{e}} \approx 10-20 \mathrm{M}\right)$. This suggests that the effects of these deformation parameters are localized quite close to the black hole. For larger viewing angles, the Doppler effect is stronger, while the gravitational redshift is the same because it does not depend on $i$. The result is that the photons with the highest energies come from smaller radii. Figure 8 shows the iron lines in Figure 7 for $i=80^{\circ}$. The impact of the deformation parameters is now stronger, and, in particular, the very high energy part of the line does depend on the value of the deformation parameters. Even a non-vanishing $\alpha_{52}$, which had an extremely weak effect for a line seen at $i=30^{\circ}$, produces some clear effects for $i=80^{\circ}$.

\section{Reflection Spectrum}

In this section, we consider the full reflection spectrum of the accretion disk and we illustrate with some examples how we can constrain the deformation parameters. We simulate observations with $N U S T A R$ and LAD/eXTP to show the constraining power of current and future X-ray missions, respectively. A detailed analysis to study the parameter degeneracy will be presented in a forthcoming paper.

We consider the case of a bright black hole binary, which is expected to be the most suitable source for this kind of test, and we set its energy flux in the $2-10 \mathrm{keV}$ range at $10^{-9} \mathrm{erg} \mathrm{s}^{-1} \mathrm{~cm}^{-2}$. The exposure time is $50 \mathrm{ks}$. The resulting total number of counts is $\sim 10^{6}$ for $N u S T A R$ and $\sim 10^{8}$ for LAD/eXTP. We simulate three observations with the extended RELXILL. The values of the input parameters are shown in Table 5. The photon index of the continuum is $\Gamma=1.6$; the spin parameter is always $a_{*}=0.8$; the emissivity profile is assumed to be a simple power law with index 3 , namely $\propto 1 / r_{\mathrm{e}}^{3}$; the ionization parameter is $\log \xi=3.1$ ( $\xi$ in units of $\mathrm{erg} \mathrm{cm} / \mathrm{s}$ ); the iron abundance is $A_{\mathrm{Fe}}=5$ (in units of Solar iron abundance); the energy cut-off of the continuum is $E_{\text {cut }}=120 \mathrm{keV}$; the reflection fraction is chosen to be 3 . In Simulation 1, we have a Kerr black hole observed from the viewing angle $i=30^{\circ}$. In Simulations 2, we have a non-Kerr black hole with the deformation parameter $\alpha_{13}=-2$ (all the other deformation parameters vanish); the inclination angle is still $i=30^{\circ}$. In Simulation 3, we have a Kerr black hole observed from the viewing angle $i=80^{\circ}$.

The last column in Table 5 shows which parameters are free and which are frozen in the fit. Since here we are merely interested in some examples to illustrate the constraints from possible observations with current and future X-ray missions, the initial values of the fit are chosen close to the actual values employed in the simulation. With $N u S T A R$, we analyze the data 

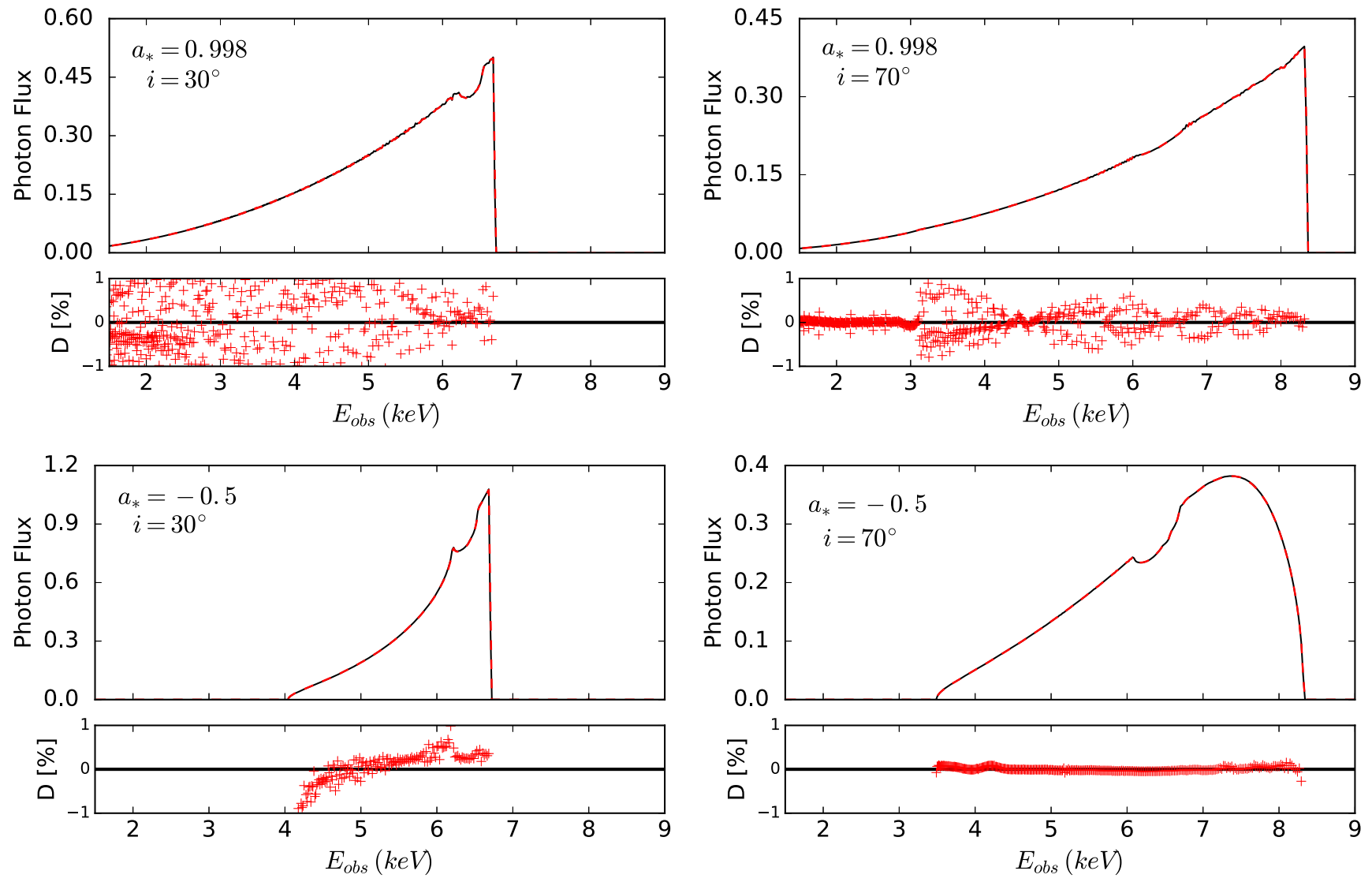

Figure 4. Comparison between single iron line shapes in the Kerr metric generated by our new code (red dashed lines) and by RELLINE (black lines) for different values of the spin parameter $a_{*}$ and the viewing angle $i$. The difference in percentage between the two lines at every energy bin is shown in the box below every panel and it is usually within $1 \%$. Top left panel: $a_{*}=0.998$ and $i=30^{\circ}$. Top right panel: $a_{*}=0.998$ and $i=70^{\circ}$. Bottom left panel: $a_{*}=-0.5$ and $i=30^{\circ}$. Bottom right panel: $a_{*}=-0.5$ and $i=70^{\circ}$.

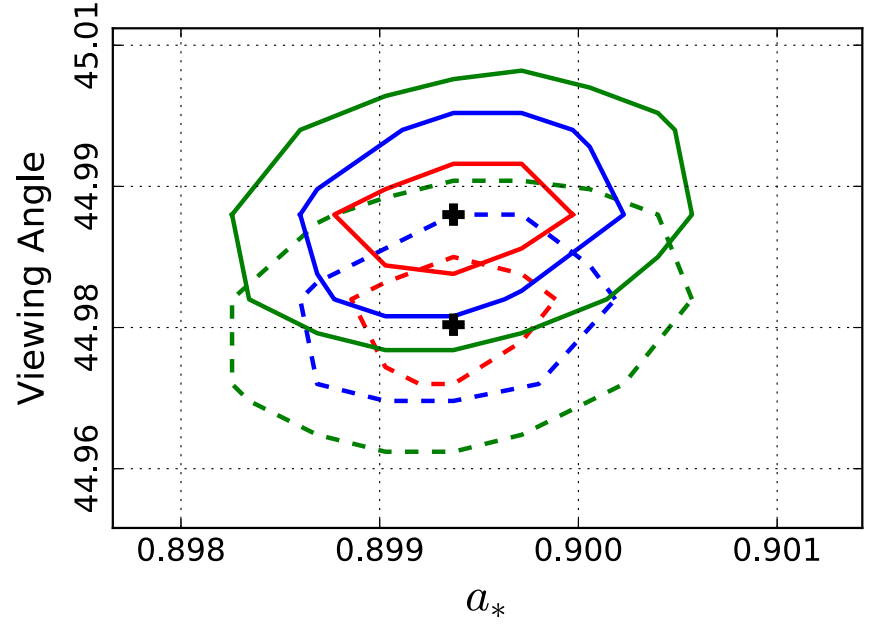

Figure 5. $\Delta \chi^{2}$ contours of the simulations described at the end of Section 5 . The simulated data have been obtained by RELLINE, plugging the spin parameter $a_{*}=0.9$ and the viewing angle $i=45^{\circ}$. The data have been fitted with the iron lines in the Kerr metric generated by the code presented in this work (dashed curves) and with the iron lines generated by RELLINE (solid curves). The black cross indicates the position of the minimum of $\chi^{2}$, while the red, blue, and green curves indicate, respectively, the $1-, 2-, 3-\sigma$ limits. See the text for more details.

in the range of 3-70 keV, while in the case of LAD/eXTP the range is $1-70 \mathrm{keV}$. Figure 9 shows the map of $\Delta \chi^{2}$ for the spin parameter and the deformation parameter $\alpha_{13}$ for Simulation 1; the left panel is the result from the simulation with NuSTAR and the right panel is that for $\mathrm{LAD} / \mathrm{eXTP}$. The red, green, and blue curves indicate, respectively, the $1-, 2-, 3-\sigma$ limits. For the simulation with LAD/eXTP, we only show the blue $3-\sigma$ contour because the allowed region is extremely thin. The gray region is not analyzed because the spacetimes there do not meet the condition on $\alpha_{13}$ in (13). The confidence contours for Simulation 2 are shown in Figure 10 and those for Simulation 3 in Figure 11.

The degeneracy between the spin and the deformation parameter $\alpha_{13}$ is clear. While this depends on the choice of the deformation parameter, so in our case $\alpha_{13}$, it is quite a common feature, especially when the deformation parameter has a strong impact on the value of the ISCO radius. In Figure 9, the contours for NuSTAR show that we could potentially find a large spin for either positive or negative deformations. The negative branch is removed with LAD/eXTP, but despite the very small uncertainty, the positive branch is still there, which means that for small inclinations the problem of degeneracy may persist despite the large effective area. In Figure 11, the inclination angle is large, which maximizes the relativistic effects and helps to break the parameter degeneracy. While this looks indeed to be the best case for NUSTAR, the problem of degeneracy persists.

The remarkable difference between the constraining power of NUSTAR and LAD/eXTP was already pointed out in $\mathrm{Ni}$ et al. (2016). We note that LAD/eXTP can potentially provide constraints that are stringent enough on the deformation 


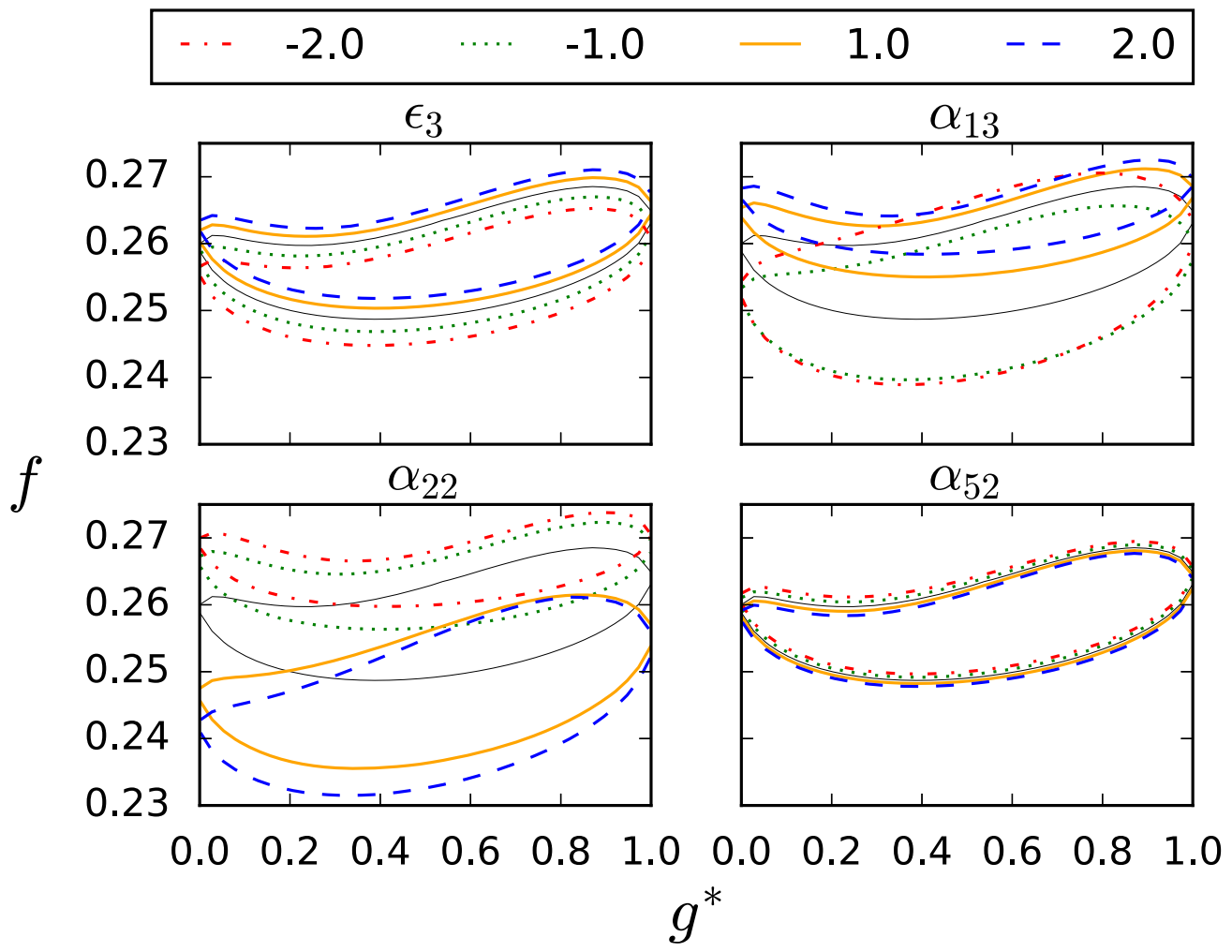

Figure 6. Impact of the deformation parameters $\epsilon_{3}, \alpha_{13}, \alpha_{22}$, and $\alpha_{52}$ on the transfer function $f$. The spacetime is described by the Johannsen metric with the spin parameter $a_{*}=0.8$. The emission radius is $r_{\mathrm{em}}=6.855$ and the viewing angle is $i=30^{\circ}$. In every plot, one of the deformation parameters assumes the values 0 (black solid line), \pm 1 , and \pm 2 , while the other deformation parameters vanish.

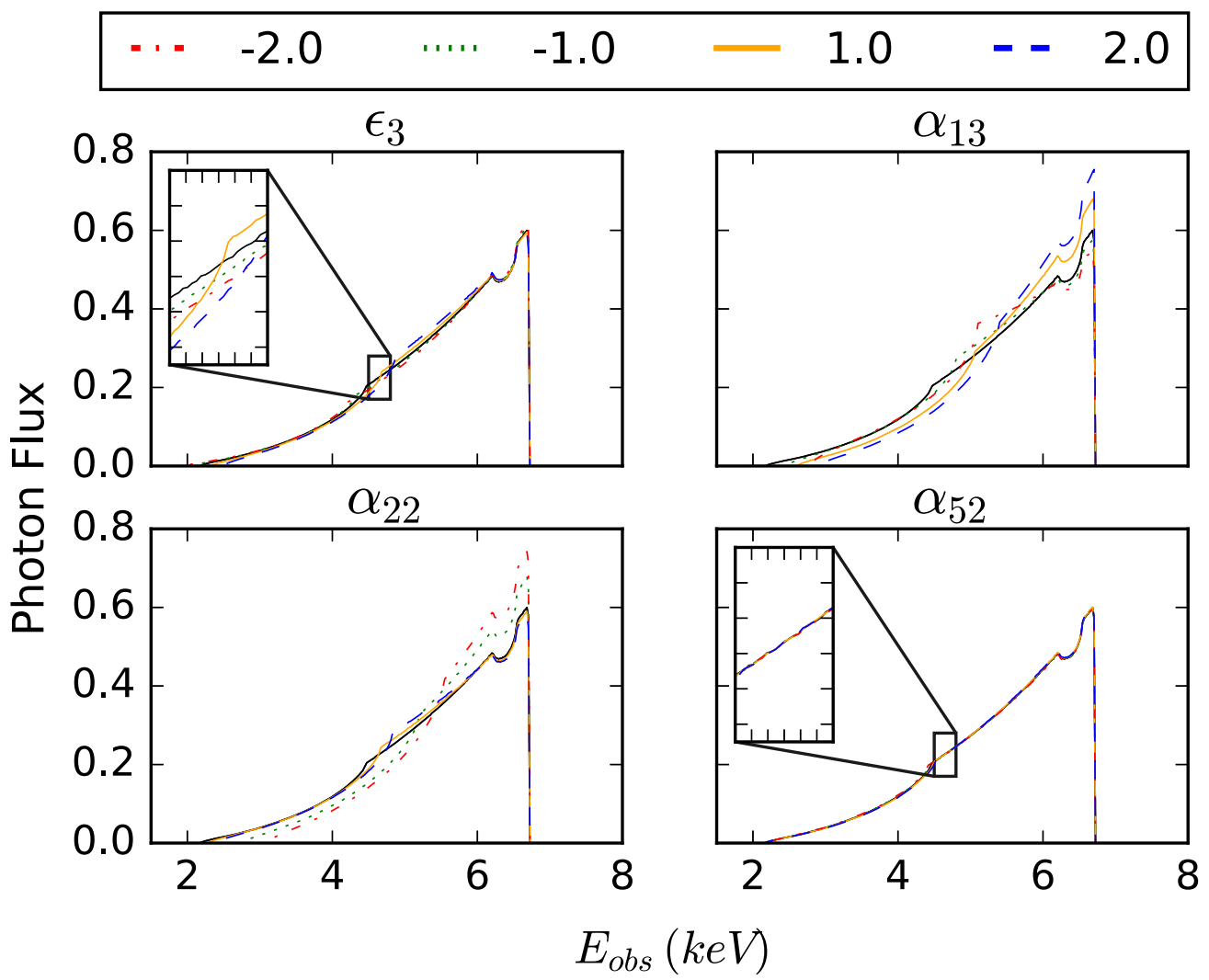

Figure 7. Impact of the deformation parameters $\epsilon_{3}, \alpha_{13}, \alpha_{22}$, and $\alpha_{52}$ on the iron line shape. The spacetime is described by the Johannsen metric with the spin parameter $a_{*}=0.8$. The viewing angle is $i=30^{\circ}$. The profile of the emissivity is modeled with a simple power law with emissivity index $q=3$, namely $I_{\mathrm{e}} \propto 1 / r_{\mathrm{e}}^{3}$. The inner edge of the disk is at the ISCO radius $r_{\text {in }}=r_{\text {ISCO }}$, and the outer edge is at $r_{\text {out }}=400$. 


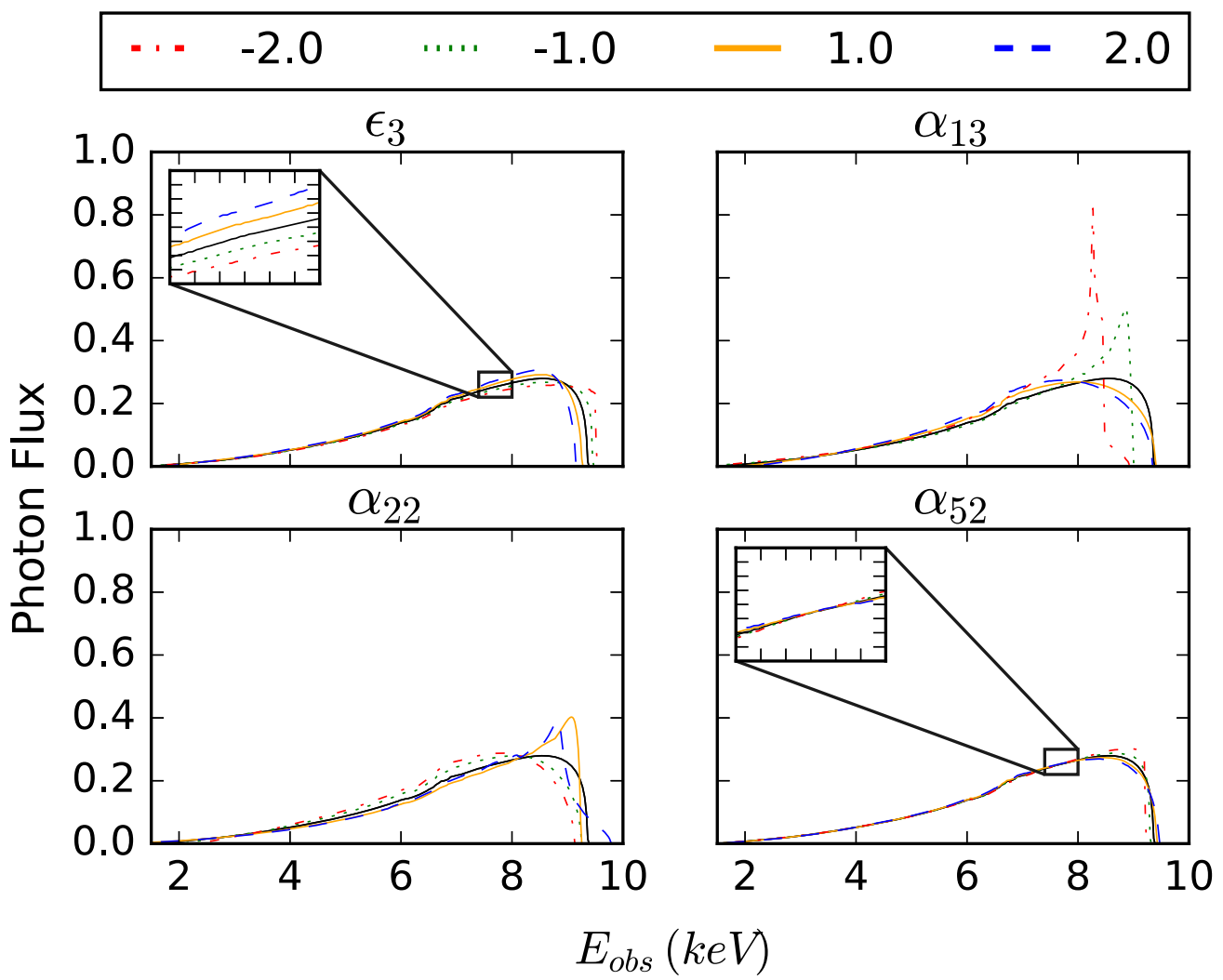

Figure 8. Same as in Figure 7, but for the viewing angle $i=80^{\circ}$.

Table 5

Summary of the simulations

\begin{tabular}{|c|c|c|c|}
\hline & Parameter & Simulation & Fit \\
\hline & Energy flux $(2-10 \mathrm{keV})$ & $10^{-9} \mathrm{erg} \mathrm{s}^{-1} \mathrm{~cm}^{-2}$ & \\
\hline & Exposure time & $50 \mathrm{ks}$ & \\
\hline & $\Gamma$ & 1.6 & free \\
\hline & $q$ & 3 & free \\
\hline & $r_{\text {in }}$ & $r_{\mathrm{ISCO}}$ & frozen \\
\hline & $r_{\text {out }}$ & 400 & frozen \\
\hline & $z$ & 0 & frozen \\
\hline & $\log \xi$ & 3.1 & free \\
\hline & $A_{\mathrm{Fe}}$ & 5 & free \\
\hline & $E_{\text {cut }}$ & $120 \mathrm{keV}$ & frozen \\
\hline & Reflection fraction & 3 & free \\
\hline & $\alpha_{13}$ & 0 & free \\
\hline Simulation 1 & $a_{*}$ & 0.8 & free \\
\hline & $i$ & $30^{\circ}$ & free \\
\hline & $\alpha_{13}$ & -2 & free \\
\hline Simulation 2 & $a_{*}$ & 0.8 & free \\
\hline & $i$ & $30^{\circ}$ & free \\
\hline & $\alpha_{13}$ & 0 & free \\
\hline Simulation 3 & $a_{*}$ & 0.8 & free \\
\hline & $i$ & $80^{\circ}$ & free \\
\hline
\end{tabular}

Summary of the values of the parameters employed in our simulations and fits. $\Gamma$ is the photon index of the power-law component, $q$ is the emissivity index, $z$ is the cosmological redshift, $\log \xi$ is the ionization parameter, and $A_{\mathrm{Fe}}$ is the iron abundance (in units of solar iron abundance).

parameters that the choice of the correct theoretical model, e.g., the choice of the form of the emissivity profile, will be crucial to get reliable constraints on the spacetime metric.

\section{Summary and Conclusions}

In this paper, we present the first X-ray reflection model for testing the spacetime metric around astrophysical black holes. Previous work suggests that the reflection method is quite a promising technique to test the Kerr black hole hypothesis with electromagnetic radiation. However, current studies employ simplified models. In the best cases, the X-ray spectrum is approximated by a power law with an iron line. Similar models can work for preliminary studies, but they are definitively inadequate to perform precise tests of general relativity in the strong gravity regime.

RELXILL is currently the most sophisticated model to fit the $\mathrm{X}$-ray reflection spectrum of black holes under the assumption that the spacetime is described by the Kerr solution. It results from the combination of the relativistic convolution model for the Kerr metric RELCONV and the reflection code for the local spectrum XILLVER. By calculating the transfer function for a generic background, we have a new relativistic convolution model to replace RELCONV. After merging our new relativistic convolution model with XILLVER, we obtain the extension of RELXILL to generic stationary, axisymmetric, and asymptotically flat black hole spacetime.

We have described our new code and the relevant formulas for the calculation of the transfer function. We have shown that our calculations reach the necessary accuracy for our tests. We have simulated some observations of a bright black hole binary with NUSTAR and LAD/eXTP to illustrate the constraining power of current and future X-ray missions. The current version of the code adopts the Johannsen metric, but it is straightforward to employ any other stationary, axisymmetric, and asymptotically flat black hole metric. Work on other non-Kerr metrics, such as that proposed in Konoplya et al. (2016), is currently 

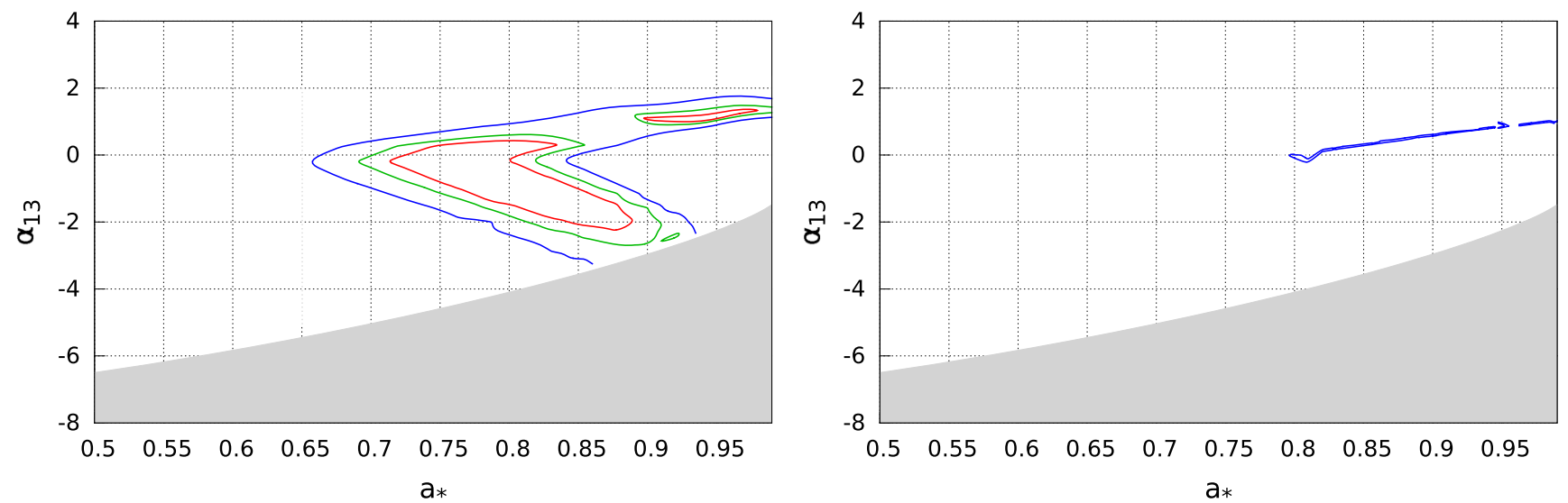

Figure 9. 1-, 2-, 3- $\sigma$ confidence contours for the spin parameter $a_{*}$ and the deformation parameter $\alpha_{13}$ from a simulated observation of a bright black hole binary with NuSTAR (left panel) and LAD/eXTP (right panel). The spacetime metric of the simulation has $\alpha_{13}=0$ (Kerr) and $a_{*}=0.8$; the viewing angle is $i=30^{\circ}$ (Simulation 1). The grayed region is outside the range prescribed for $\alpha_{13}$ in (13) and therefore we restrict our analysis to the regions above. See the text for more details.
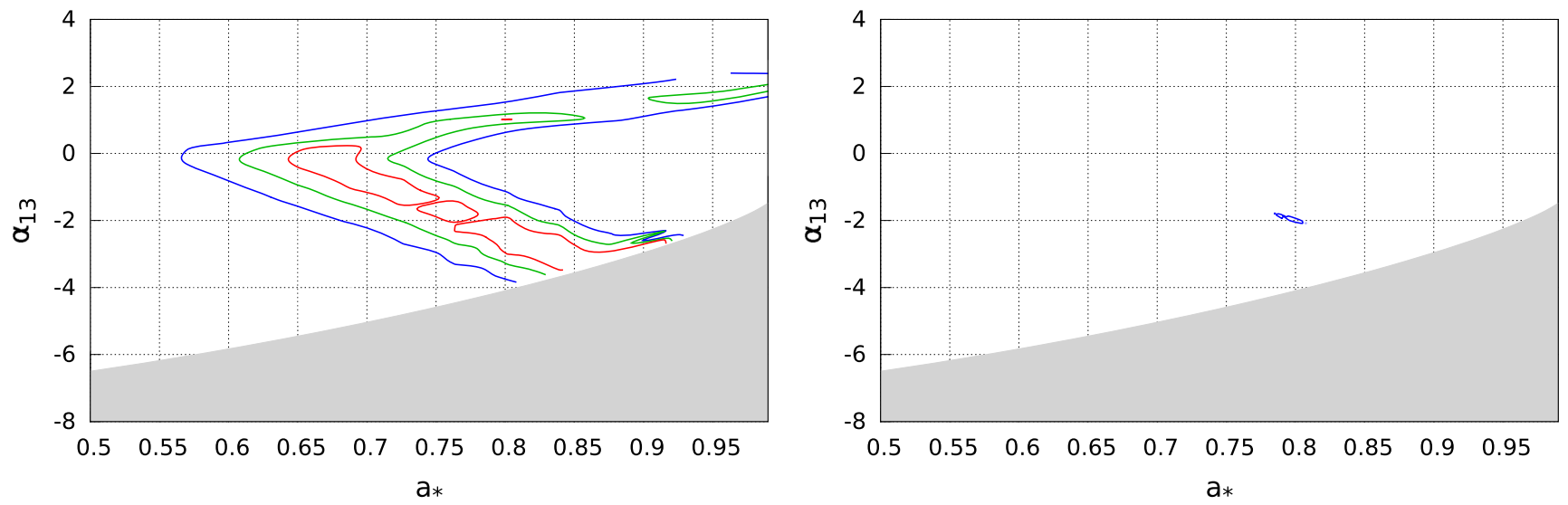

Figure 10. Same as in Figure 9 for $\alpha_{13}=-2, a_{*}=0.8$, and $i=30^{\circ}$ (Simulation 2). See the text for more details.
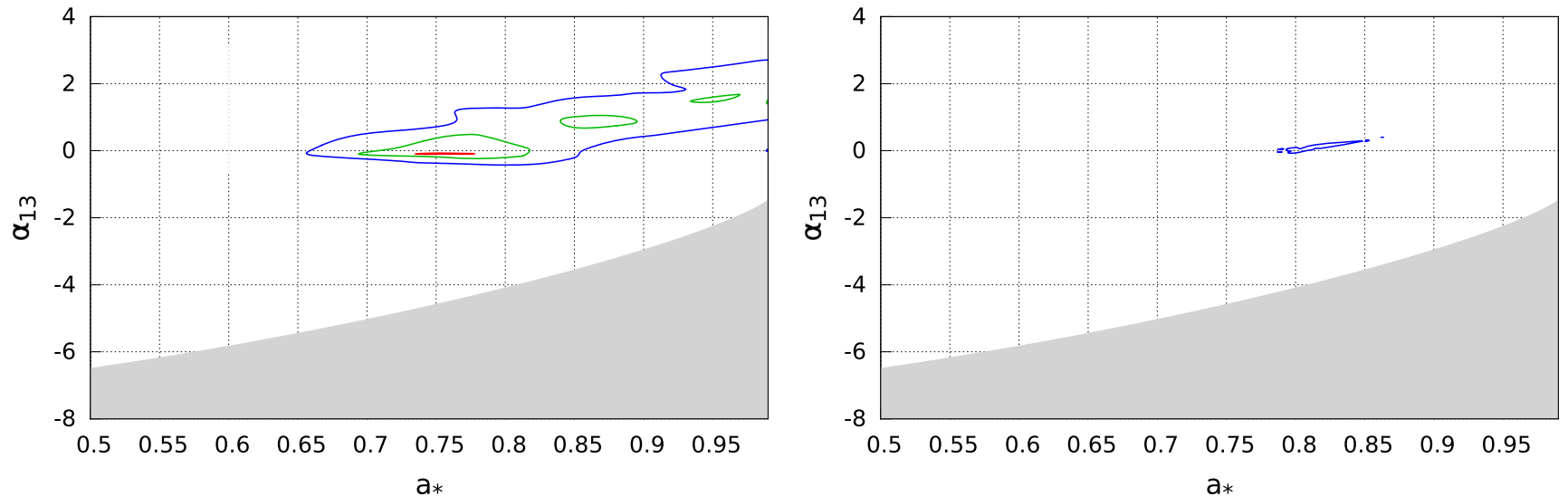

Figure 11. Same as in Figure 9 for $\alpha_{13}=0$ (Kerr), $a_{*}=0.8$, and $i=80^{\circ}$ (Simulation 3). See the text for more details.

underway. In a forthcoming paper, we will apply our new model to a specific source for constraining the deformation parameters of the Johannsen metric from available X-ray data.

We thank Jiachen Jiang for useful discussions and suggestions. C.B. and S.N. were supported by the NSFC (grants U1531117 and 11305038), Fudan University (Grant No. IDH1512060), and the
Thousand Young Talents Program. C.B. also acknowledges the support from the Alexander von Humboldt Foundation. A.C.-A. acknowledges funding from the Fundación Universitaria Konrad Lorenz (Project 5INV1) and thanks the Department of Physics at Fudan University for hospitality during his visit. JAG acknowledges the support of a CGPS grant from the Smithsonian Institution. 


\section{Appendix Calculations of the Transfer Function}

In order to calculate the transfer function in Equation (3), we have to map the emission points in the disk onto the image plane of the distant observer. This can be achieved by calculating the photon trajectories connecting the emission points to the detection points.

\section{A.1. Photon Initial Conditions}

The first step is to write the photon initial conditions in the image plane of the distant observer (Johannsen \& Psaltis 2010; Bambi 2012). Let us consider a black hole surrounded by an accretion disk and an observer at the distant $D$ from the black hole and with the viewing angle $i$, as sketched in Figure 12. The image plane of the distant observer is provided with a system of Cartesian coordinates $(X, Y, Z)$. Another system of Cartesian coordinates $(x, y, z)$ is centered at the black hole. The two Cartesian coordinates are related by

$$
\begin{aligned}
& x=D \sin i-Y \cos i+Z \sin i, \\
& y=X, \\
& z=D \cos i+Y \sin i+Z \cos i .
\end{aligned}
$$

Let us assume that the black hole metric is expressed in spherical-like coordinates. Far from the compact object, the spatial coordinates reduce to the usual spherical coordinates in flat spacetime and they are related to $(x, y, z)$ by

$$
\begin{aligned}
r & =\sqrt{x^{2}+y^{2}+z^{2}}, \\
\theta & =\arccos \left(\frac{z}{r}\right), \\
\phi & =\arctan \left(\frac{y}{x}\right) .
\end{aligned}
$$

Let us consider a photon at the position $\left(X_{0}, Y_{0}, 0\right)$ and with 3-momentum $\boldsymbol{k}_{0}=-k_{0} \hat{Z}$ perpendicular to the image plane. The initial conditions for the photon position are

$$
\begin{aligned}
t_{0} & =0, \\
r_{0} & =\sqrt{X_{0}^{2}+Y_{0}^{2}+D^{2}}, \\
\theta_{0} & =\arccos \frac{Y_{0} \sin i+D \cos i}{r_{0}}, \\
\phi_{0} & =\arctan \frac{X_{0}}{D \sin i-Y_{0} \cos i} .
\end{aligned}
$$

The photon 4-momentum is $k^{\mu}=\left(\partial x^{\mu} / \partial \tilde{x}^{\alpha}\right) \tilde{k}^{\alpha}$, where $\tilde{k}^{\alpha}=\left(k_{0}, 0,0,-k_{0}\right)$ is the photon 4-momentum in the Cartesian coordinates, and we find

$$
\begin{aligned}
k_{0}^{r} & =-\frac{D}{r_{0}}\left|\boldsymbol{k}_{0}\right|, \\
k_{0}^{\theta} & =\frac{\cos i-\left(Y_{0} \sin i+D \cos i\right) \frac{D}{r_{0}^{2}}}{\sqrt{X_{0}^{2}+\left(D \sin i-Y_{0} \cos i\right)^{2}}}\left|\boldsymbol{k}_{0}\right|, \\
k_{0}^{\phi} & =\frac{X_{0} \sin i}{X_{0}^{2}+\left(D \sin i-Y_{0} \cos i\right)^{2}}\left|\boldsymbol{k}_{0}\right| .
\end{aligned}
$$

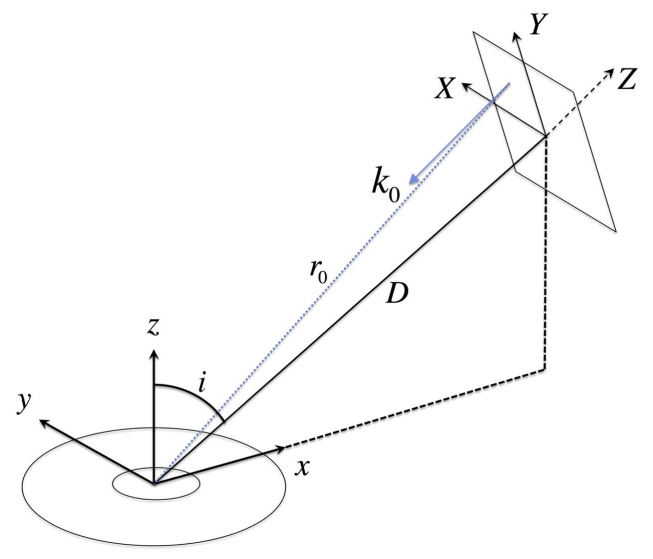

Figure 12. Cartesian coordinates $(x, y, z)$ are centered at the black hole, while the Cartesian coordinates $(X, Y, Z)$ are for the image plane of the distant observer, which is located at the distant $D$ from the black hole and with an inclination angle $i$.

$k_{0}^{t}$ can be obtained from the condition $g_{\mu \nu} k^{\mu} k^{\nu}=0$ with the metric tensor of a flat spacetime, so

$$
k_{0}^{t}=\sqrt{\left(k_{0}^{r}\right)^{2}+r_{0}^{2}\left(k_{0}^{\theta}\right)^{2}+r_{0}^{2} \sin ^{2} \theta_{0}\left(k_{0}^{\phi}\right)^{2}} .
$$

\section{A.2. Photon Trajectory}

With the photon initial conditions (25)-(27), we can integrate the geodesic equations backward in time from any detection point $\left(X_{0}, Y_{0}, 0\right)$ in the image plane of the distant observer to the emission point in the disk:

$$
\frac{d^{2} x^{\mu}}{d \tau^{2}}+\Gamma_{\nu \rho}^{\mu} \frac{d x^{\nu}}{d \tau} \frac{d x^{\rho}}{d \tau}=0,
$$

where $\tau$ is an affine parameter. In the case of the Kerr metric, it is not necessary to directly integrate the geodesic equations and the calculations are thus different (Cunningham 1975; Speith et al. 1995). In the Kerr metric in Boyer-Lindquist coordinates, the equations of motion are separable and we can restrict the attention to the motion in the $(r, \theta)$ plane; the corresponding equations can be solved in terms of elliptic integrals.

\section{A.3. Accretion Disk}

The integration of the geodesic equations stops when the photon either hits the accretion disk or misses it. In the latter case, the photon either hits the black hole or crosses the equatorial plane between the black hole and the inner edge of the disk. In our model, only the primary image of the accretion disk is take into account, and there is no emission between the black hole and the inner edge of the disk. The inner edge of the disk is assumed at the ISCO radius and, for a generic stationary, axisymmetric, and asymptotically flat spacetime can be inferred as follows. We write the line element in the canonical form, namely,

$$
d s^{2}=g_{t t} d t^{2}+2 g_{t \phi} d t d \phi+g_{r r} d r^{2}+g_{\theta \theta} d \theta^{2}+g_{\phi \phi} d \phi^{2},
$$

where the metric coefficients are independent of $t$ and $\phi$. The motion of a test particle in the metric background is governed by the Lagrangian

$$
\mathcal{L}=\frac{1}{2} g_{\mu \nu} \dot{x}^{\mu} \dot{x}^{\nu}
$$


where $=d / d \tau$. Since the metric is independent of the coordinates $t$ and $\phi$, we have two constants of motion, namely the specific energy at infinity $E$ and the axial component of the specific angular momentum at infinity $L_{z}$ :

$$
\begin{gathered}
\frac{d}{d \tau} \frac{\partial \mathcal{L}}{\partial \dot{t}}-\frac{\partial \mathcal{L}}{\partial t}=0 \Rightarrow p_{t} \equiv \frac{\partial \mathcal{L}}{\partial \dot{t}}=g_{t t} \dot{t}+g_{t \phi} \dot{\phi}=-E, \\
\frac{d}{d \tau} \frac{\partial \mathcal{L}}{\partial \dot{\phi}}-\frac{\partial \mathcal{L}}{\partial \phi}=0 \Rightarrow p_{\phi} \equiv \frac{\partial \mathcal{L}}{\partial \dot{\phi}}=g_{t \phi} \dot{t}+g_{\phi \phi} \dot{\phi}=L_{z} .
\end{gathered}
$$

The term "specific" is used to indicate that $E$ and $L_{z}$ are, respectively, the energy and angular momentum per unit restmass. Equations (31) and (32) can be solved to find the $t$ - and the $\phi$-component of the 4-velocity of the test particle

$$
\dot{t}=\frac{E g_{\phi \phi}+L_{z} g_{t \phi}}{g_{t \phi}^{2}-g_{t t} g_{\phi \phi}}, \quad \dot{\phi}=-\frac{E g_{t \phi}+L_{z} g_{t t}}{g_{t \phi}^{2}-g_{t t} g_{\phi \phi}} .
$$

The accretion disk is described by the Novikov-Thorne model (Novikov et al. 1973; Page \& Thorne 1974). The disk is in the equatorial plane perpendicular to the black hole spin. The particles of the gas follow nearly geodesic, equatorial, and circular orbits. We write the geodesic equations as

$$
\frac{d}{d \tau}\left(g_{\mu \nu} \dot{x}^{\nu}\right)=\frac{1}{2}\left(\partial_{\mu} g_{\nu \rho}\right) \dot{x}^{\nu} \dot{x}^{\rho} .
$$

Since $\dot{r}=\dot{\theta}=\ddot{r}=0$ for equatorial circular orbits, the radial component of Equation (34) reduces to

$$
\left(\partial_{r} g_{t t}\right) \dot{t}^{2}+2\left(\partial_{r} g_{t \phi}\right) \dot{t} \dot{\phi}+\left(\partial_{r} g_{\phi \phi}\right) \dot{\phi}^{2}=0 .
$$

The angular velocity $\Omega=\dot{\phi} / \dot{t}$ is

$$
\Omega=\frac{-\partial_{r} g_{t \phi} \pm \sqrt{\left(\partial_{r} g_{t \phi}\right)^{2}-\left(\partial_{r} g_{t t}\right)\left(\partial_{r} g_{\phi \phi}\right)}}{\partial_{r} g_{\phi \phi}},
$$

where the upper (lower) sign refers to corotating (counterrotating) orbits, namely orbits with angular momentum parallel (antiparallel) to the spin of the central object.

From $g_{\mu \nu} \dot{x}^{\mu} \dot{x}^{\nu}=-1$ with $\dot{r}=\dot{\theta}=0$, we can write

$$
\dot{t}=\frac{1}{\sqrt{-g_{t t}-2 \Omega g_{t \phi}-\Omega^{2} g_{\phi \phi}}} .
$$

Equation (31) becomes

$$
\begin{aligned}
E & =-\left(g_{t t}+\Omega g_{t \phi}\right) \dot{t} \\
& =-\frac{g_{t t}+\Omega g_{t \phi}}{\sqrt{-g_{t t}-2 \Omega g_{t \phi}-\Omega^{2} g_{\phi \phi}}} .
\end{aligned}
$$

In the same way, Equation (32) becomes

$$
\begin{aligned}
L_{z} & =\left(g_{t \phi}+\Omega g_{\phi \phi}\right) \dot{t} \\
& =\frac{g_{t \phi}+\Omega g_{\phi \phi}}{\sqrt{-g_{t t}-2 \Omega g_{t \phi}-\Omega^{2} g_{\phi \phi}}} .
\end{aligned}
$$

From $g_{\mu \nu} \dot{x}^{\mu} \dot{x}^{\nu}=-1$ and with the use of Equation (33), we can now write

$$
g_{r r} \dot{r}^{2}+g_{\theta \theta}^{2} \dot{\theta}^{2}=V_{\mathrm{eff}}\left(r, \theta, E, L_{z}\right),
$$

where $V_{\text {eff }}\left(r, \theta, E, L_{z}\right)$ is the effective potential of the test particle with energy $E$ and axial component of the angular momentum $L_{z}$

$$
V_{\mathrm{eff}}=\frac{E^{2} g_{\phi \phi}+2 E L_{z} g_{t \phi}+L_{z}^{2} g_{t t}}{g_{t \phi}^{2}-g_{t t} g_{\phi \phi}}-1 .
$$

In the case of equatorial circular orbits, $\dot{r}=\dot{\theta}=\ddot{r}=\ddot{\theta}=0$ and therefore $V_{\text {eff }}=\partial_{r} V_{\text {eff }}=\partial_{\theta} V_{\text {eff }}=0$. The orbit is radially (vertically) stable if $\partial_{r}^{2} V_{\text {eff }}<0 \quad\left(\partial_{\theta}^{2} V_{\text {eff }}<0\right)$ and radially (vertically) unstable if $\partial_{r}^{2} V_{\text {eff }}>0\left(\partial_{\theta}^{2} V_{\text {eff }}>0\right)$. The ISCO radius $r_{\text {ISCO }}$ is given by

$$
\partial_{r}^{2} V_{\text {eff }}=0 \text { or } \partial_{\theta}^{2} V_{\text {eff }}=0 \Rightarrow r=r_{\text {ISCO }} .
$$

\section{A.4. Redshift Factor and Emission Angle}

Once we know the emission point in the disk, we can evaluate the redshift factor $g$ and the emission angle $\vartheta_{\mathrm{e}}$. The redshift factor $g$ is

$$
g=\frac{\nu_{\mathrm{o}}}{\nu_{\mathrm{e}}}=\frac{-u_{\mathrm{o}}^{\mu} k_{\mu}}{-u_{\mathrm{e}}^{\nu} k_{\nu}},
$$

where $u_{\mathrm{o}}^{\mu}=(1,0,0,0)$ is the 4-velocity of the distant observer, $k^{\mu}$ is the 4-momentum of the photon, and $u_{\mathrm{e}}^{\nu}=u_{\mathrm{e}}^{t}(1,0,0, \Omega)$ is the 4-velocity of the particles of the gas. $u_{\mathrm{e}}^{t}=\dot{t}$, which is given by Equation (37). Plugging Equation (37) into Equation (43), we obtain

$$
g=\frac{\sqrt{-g_{t t}-2 g_{t \phi} \Omega-g_{\phi \phi} \Omega^{2}}}{1-\lambda \Omega},
$$

where $\lambda=-k_{\phi} / k_{t}$ is a constant of motion along the photon trajectory and can be evaluated from the initial conditions.

If the local spectrum depends on the emission angle $\vartheta_{\mathrm{e}}$, the latter must be rewritten in terms of the emission radius and redshift factor. The normal of the disk is

$$
n^{\mu}=\left.\left(0,0, \sqrt{g^{\theta \theta}}, 0\right)\right|_{r_{\mathrm{e}}, \theta_{\mathrm{e}}=\pi / 2},
$$

and therefore the cosine of the emission angle $\vartheta_{\mathrm{e}}$ is

$$
\cos \vartheta_{\mathrm{e}}=\left.\frac{n^{\mu} k_{\mu}}{u_{\mathrm{e}}^{\nu} k_{\nu}}\right|_{\mathrm{e}}=\sqrt{g^{\theta \theta}} \frac{\sqrt{-g_{t t}-2 g_{t \phi} \Omega-g_{\phi \phi} \Omega^{2}}}{1-\lambda \Omega} \frac{k_{\theta}}{k_{t}},
$$

where $k_{\theta}$ is the $\theta$-component of the 4-momentum of the photon at the point of emission in the disk and, in the general case, it is determined at the end of the geodesic integration.

At the end of the integration of the photon trajectory, we have $r_{\mathrm{e}}=r_{\mathrm{e}}(X, Y), g=g(X, Y)$, and $\vartheta_{\mathrm{e}}=\vartheta_{\mathrm{e}}(X, Y)$. From the first two relations, it is possible to numerically compute the Jacobian in the transfer function

$$
\left|\frac{\partial(X, Y)}{\partial\left(g^{*}, r_{\mathrm{e}}\right)}\right|=\left(g_{\max }-g_{\min }\right)\left|\frac{\partial X}{\partial g} \frac{\partial Y}{\partial r_{\mathrm{e}}}-\frac{\partial X}{\partial r_{\mathrm{e}}} \frac{\partial Y}{\partial g}\right| .
$$

This completes the calculations of the transfer function $f$ for a specific background metric. If we know the local spectrum of the radiation $I_{\mathrm{e}}$, we can obtain the observed flux via Equation (2).

\section{References}

Bambi, C. 2012, ApJ, 761, 174

Bambi, C. 2013a, PhRvD, 87, 023007

Bambi, C. 2013b, JCAP, 8, 055 
Bambi, C. 2017, RvMP, 89, 025001

Bambi, C., \& Barausse, E. 2011, ApJ, 731, 121

Bambi, C., Dolgov, A. D., \& Petrov, A. A. 2009, JCAP, 9, 013

Bambi, C., Jiang, J., \& Steiner, J. F. 2016, CQGra, 33, 064001

Bambi, C., \& Malafarina, D. 2013, PhRvD, 88, 064022

Bambi, C., Malafarina, D., \& Tsukamoto, N. 2014, PhRvD, 89, 127302

Bambi, C., \& Nampalliwar, S. 2016, EL, 116, 30006

Barausse, E., Cardoso, V., \& Pani, P. 2014, PhRvD, 89, 104059

Brenneman, L. W., \& Reynolds, C. S. 2006, ApJ, 652, 1028

Cárdenas-Avendaño, A., Jiang, J., \& Bambi, C. 2016, PhLB, 760, 254

Carter, B. 1971, PhRvL, 26, 331

Chruściel, P. T., Costa, J. L., \& Heusler, M. 2012, LRR, 15, 7

Cunningham, C. T. 1975, ApJ, 202, 788

Czerny, B., Hryniewicz, K., Nikołajuk, M., \& Sądowski, A. 2011, MNRAS, 415, 2942

Dauser, T., Garcia, J., Wilms, J., et al. 2013, MNRAS, 430, 1694

Dauser, T., Wilms, J., Reynolds, C. S., \& Brenneman, L. W. 2010, MNRAS, 409, 1534

Done, C., Jin, C., Middleton, M., \& Ward, M. 2013, MNRAS, 434, 1955

Dvali, G., \& Gomez, C. 2013a, ForPh, 61, 742

Dvali, G., \& Gomez, C. 2013b, PhLB, 719, 419

Dyson, F. W., Eddington, A. S., \& Davidson, C. 1920, RSPTA, 220, 291

Fabian, A. C., Rees, M. J., Stella, L., \& White, N. E. 1989, MNRAS, 238, 729

Fabian, A. C., \& Ross, R. R. 2010, SSRv, 157, 167

García, J., Dauser, T., Lohfink, A., et al. 2014, ApJ, 782, 76

García, J., Dauser, T., Reynolds, C. S., et al. 2013, ApJ, 768, 146

García, J., \& Kallman, T. R. 2010, ApJ, 718, 695

García, J., Kallman, T. R., Witthoeft, M., et al. 2009, ApJS, 185, 477

García, J., Mendoza, C., Bautista, M. A., et al. 2005, ApJS, 158, 68

Giddings, S. B. 2014, PhRvD, 90, 124033

Herdeiro, C. A. R., \& Radu, E. 2014, PhRvL, 112, 221101

Jiang, J., Bambi, C., \& Steiner, J. F. 2015a, JCAP, 5, 025

Jiang, J., Bambi, C., \& Steiner, J. F. 2015b, ApJ, 811, 130

Jiang, J., Bambi, C., \& Steiner, J. F. 2016, PhRvD, 93, 123008
Johannsen, T. 2013, PhRvD, 88, 044002

Johannsen, T. 2014, PhRvD, 90, 064002

Johannsen, T. 2016, CQGra, 33, 124001

Johannsen, T., \& Psaltis, D. 2010, ApJ, 718, 446

Johannsen, T., \& Psaltis, D. 2013, ApJ, 773, 57

Kallman, T., \& Bautista, M. 2001, ApJS, 133, 221

Kallman, T. R., Palmeri, P., Bautista, M. A., Mendoza, C., \& Krolik, J. H. 2004, ApJS, 155, 675

Kong, L., Li, Z., \& Bambi, C. 2014, ApJ, 797, 78

Konoplya, R., Rezzolla, L., \& Zhidenko, A. 2016, PhRvD, 93, 064015

Konoplya, R., \& Zhidenko, A. 2016, PhLB, 756, 350

Lu, Y., \& Torres, D. F. 2003, IJMPD, 12, 63

Lund, E., Bugge, L., Gavrilenko, I., \& Strandlie, A. 2009, JInst, 4, 04001

McClintock, J. E., Narayan, R., \& Steiner, J. F. 2014, SSRv, 183, 295

Mignemi, S., \& Stewart, N. R. 1993, PhRvD, 47, 5259

Ni, Y., Zhou, M., Cárdenas-Avendaño, A., et al. 2016, JCAP, 7, 049

Novikov, I. D., \& Thorne, K. S. 1973, in Black Holes, ed. C. De Witt \&

B. De Witt (New York: Gordon and Breach), 343

Page, D. N., \& Thorne, K. S. 1974, ApJ, 191, 499

Price, R. H. 1972, PhRvD, 5, 2419

Psaltis, D., Perrodin, D., Dienes, K. R., \& Mocioiu, I. 2008, PhRvL, 100, 119902

Reynolds, C. S. 2014, SSRv, 183, 277

Robinson, D. C. 1975, PhRvL, 34, 905

Schee, J., \& Stuchlík, Z. 2009, GReGr, 41, 1795

Shafee, R., McClintock, J. E., Narayan, R., et al. 2006, ApJL, 636, L113

Speith, R., Riffert, H., \& Ruder, H. 1995, CoPhC, 88, 109

Torres, D. F. 2002, NuPhB, 626, 377

Will, C. M. 2014, LRR, 17, 4

Yagi, K., \& Stein, L. C. 2016, CQGra, 33, 054001

Yunes, N., \& Siemens, X. 2013, LRR, 16, 9

Zhang, S. N., Cui, W., \& Chen, W. 1997, ApJL, 482, L155

Zhang, S. N., Feroci, M., Santangelo, A., et al. 2016, Proc. SPIE, 9905 , 99051Q 